Sharif University of Technology
Scientia Iranica
SCIENTIA
IRAN ICAA
http://scientiairanica.sharif.edu

\title{
An equation for estimating the maximum normal strain of buried steel pipes in bend area under propagating waves in sands
}

\author{
E. Mohammadi ${ }^{a}$ and F. Behnamfar ${ }^{\mathrm{b}, *}$ \\ a. Freelance Structural Engineer, Shahrekord, Chaharmahal and Bakhtiari 8815968717, Iran. \\ b. Department of Civil Engineering, Isfahan University of Technology, Isfahan 8415683111, Iran. \\ Received 24 March 2020; received in revised form 16 May 2021; accepted 19 July 2021
}

\author{
KEYWORDS \\ Buried pipe; \\ Bend; \\ Wave propagation; \\ Continuum; \\ Semi-analytical \\ equation.
}

\begin{abstract}
The possible vulnerability of pipelines under propagating waves, especially in bend areas, emphasizes the need for much research in this context. Although beam and beam-shell hybrid models are usually used in the studies conducted in this field, the current study employs a continuum model. To this end, the bent pipe was modeled using shell elements, and suitable boundary conditions were taken into account to simulate the infinite length of the pipe away from the bend. The soil around the pipe was also modeled using 3D elements in compliance with the Mohr-Coulomb rule of behavior. In addition, equivalent boundary conditions were imposed on the boundaries of the soil domain where it is truncated. The properties of the pipe-soil model varied so that they were investigated under seven different ground motions, and the maximum axial strain was calculated in the bend area. The effects of different parameters including incidence angle of seismic waves, bend angle, ratio of pipe diameter to wall thickness, and physical properties of soil were also evaluated. Based on the results of analysis and regression, an equation was proposed for estimating the maximum normal strain of the buried pipes in the bend area with good accuracy.

(C) 2022 Sharif University of Technology. All rights reserved.
\end{abstract}

\section{Introduction}

Pipelines are used over long distances and within different soils, and they are subject to different detrimental factors such as different ground motions and faulting in their route. In addition, they should be bent in certain locations for re-routing purposes. Given the three-dimensional nature of geometry in a bend, the state of stress under the imposed loads and earthquake motions is complicated in bent locations; hence, it

*. Corresponding author.

E-mail addresses: ehsanm9068@gmail.com (E.

Mohammadi); farhad@iut.ac.ir (F. Behnamfar)

doi: $10.24200 /$ sci. 2021.55694 .4356 cannot be predicted by commonly available equations of solid mechanics.

Newmark and his colleagues are pioneers of conducting research on the seismic behavior of buried pipes [1]. They presented an approximate method for studying the behavior of buried pipes subjected to wave propagation. However, they disregarded the effects of inertial force and pipe-soil interaction and assumed equal strains for ground and pipe.

Sakurai and Takahashi studied pipe response to wave propagation [2]. They examined the role of inertia force in response to straight pipes and concluded that it was not significant compared to other factors. O'Rourke and El Hmadi studied the effect of seismic waves on buried pipelines [3]. They modified a study already conducted by Sakurai and Takahashi with the 
assumption of equal strains for pipe and ground. Up to now, most of the analyses were done using a beamon-Winkler-springs model in which a pipe was modeled with beam elements placed along its axis. Meanwhile, Takada and Tanabe [4], Takada and Higashi [5], Stamos and Beskos [6], Datta [7], Takada and Katagiri [8], and Kouretzis et al. [9] used a shell model for the pipe. In this approach, the pipe was modeled using cylindrical thin shell elements that made it possible to determine different components of the pipe stresses and strains. Azizkandi et al. [10] investigated the effects of soil relative density and toe condition on the interaction between two similar piles in sandy soils using centrifuge modeling. They concluded that the relative density of soil played an important role in the coefficient of interaction between adjacent piles. Therefore, they proposed a modification factor to consider the soil relative density. Khaksar et al. [11] employed a combination of centrifuge and numerical modeling methods to overcome the geometrical limitation of the small-scale physical modeling. The developed numerical model was also used to develop a spring boundary condition, which was supposed to represent the response of the omitted parts of the pipeline. Then, a centrifuge test was conducted by imposing the end boundary condition on a larger scale for the specimen.

In recent years, several novel numerical methods have been proposed to model the wave propagation problem, including the general particle dynamics, Extended Finite Element Method (XFEM), peridynamics procedure, and phase field method. They were briefly reviewed in the same order.

Zhou et al. [12] proposed a new meshless method, general particle dynamics, to simulate the samples of rock-like brittle heterogeneous material containing preexisting flaws. Their method was to numerically simulate the initiation, growth, and coalescence of cracks. The novelty here was that the preexisting flaws were simulated by empty particles. The particle was removed when its stresses could satisfy the strength criterion, and the growth path of cracks was captured through the sequence of such damaged particles. Later, they extended it to the three-dimensional case [13].

Zhou and Yang [14] developed a multi-scale numerical model in which the coordination between the internal boundary (such as cracks and holes) and meshes was not necessary for simulating the damage evolution of crack-weakened rock masses based on XFEM. In their method, the contact constraint on crack surfaces was embedded within the total stiffness matrix using penalty method, and the path of crack propagation and stress fields were determined through iterative computations. Zhou et al. [15] proposed a node-scheme method to model the frictional contact problem within the framework of XFEM. In this regard, they considered the stress redistribution of the local part of the crack surface with the advantage of solving the locking phenomenon in the numerical simulation of the frictional contact problem. Chen and Zhou [16] introduced an enhanced XFEM for propagation of complex branched cracks through coupling of the phantom node method with the mesh cut technique in the framework of the XFEM. Instead of the constant crack propagation length, they introduced a novel crack propagation scheme into the XFEM, in which the crack propagation length was a variable determined by two trial calculations.

Wang et al. [17] proposed a new method for simulating the initiation, propagation, and coalescence of the pre-existing flaws in rocks subjected to compressive loads by incorporating the maximum tensile stress and Mohr-Coulomb criteria into the extended non-ordinary state-based peridynamics. They succeeded to model and distinguish wing cracks, oblique secondary cracks, quasi-coplanar secondary cracks, and anti-wing cracks using their proposed numerical method. In a conjugated bond-pair-based peridynamic method proposed by Wang et al. [18], initiation and propagation of cracks were modeled in brittle solids. In this method, the interacting forces between two material points within one horizon were related to not only the stretch of the bond, but the rotation of the conjugated bond angles, which overcame the limitation of the fixed Poisson's ratio in the regular bond-based peridynamics.

Zhou et al. [19] presented an insight into fracture mechanism of ring Brazilian disc specimens (used for determining the tensile strength and mixed mode fracture toughness) using phase field method. They evaluated the effects of aperture ratio and crack inclination angle on the failure process and peak load of the disc specimens. Wang and Zhou [20] developed a staggered scheme for phase field modeling of brittle fractures to simulate mixed mode fracturing of disc-type specimens. The crack propagation process and load-displacement curves of each type of disc specimen were investigated using the mentioned method. Moreover, the effects of specimen geometry and loading mode on the crack propagation process and load-displacement curves were studied.

The research conducted by Shah and Chu was among the first on the pipe bend behavior [21]. They studied rigid and flexible bends and obtained closedform solutions. Ogawa and Koike [22] examined buried pipelines and obtained a design formula for the strains at bend. Mclaughlin and O'Rourke [23] and Lee et al. [24] studied strain at bend under propagating waves and examined different response parameters. Hatzigeorgiou and Beskos [25] studied the long tunnels in a similar direction where analysis of axial and bending deformations of tunnels was done using a three-dimensional model. Saberi et al. [26] (2013) examined the seismic behavior of buried steel pipes at 
the bend point under propagating waves using beam and beam-shell hybrid models. They also evaluated the effect of different factors on pipe strains at bend and derived a semi-analytical model for calculation.

A majority of investigations of the behavior of buried pipes are related to two-dimensional quasistatic analysis. Among the conducted studies, only a few cases considered bent pipes. Moreover, in the few studies on the bent pipes, mostly the beam or shell models on Winkler springs was used while the pipe was actually embedded in a three-dimensional medium. In this regard, this study puts its main focus on the three-dimensional dynamic analysis of a soil medium containing a buried bent pipe. For this purpose, an accurate model for the buried pipe and soil was proposed. Incidence angle of the seismic waves, bend angle, ratio of pipe diameter to wall thickness, and physical properties of soil are the key parameters analyzed in this study. Through regression analysis, an equation was proposed to estimate the normal strain of the pipe in the bend area. This is deemed to be the main achievement of this study.

Seismically induced permanent ground deformations are known to be responsible for major underground pipe failures. In this respect, this study aims to retrieve the cases where the pipe is not safe in the bend area solely under seismic waves. The outcome can remove the false sense of safety whenever the ground condition does not expose a permanent deformation hazard.

\section{Material properties}

The pipe used in this study was made of steel and it followed Von-Mises yield criterion for isotropic hardening. The pipe properties were extracted from API-5L X65 [27] (2000), as presented in Table 1. The soil around the pipe is a sandy medium categorized in three different states of loose, medium, and dense sands. Table 2 presents the properties of the soil types [28]. It should be noted that both $E$ and $V_{s}$ values mentioned in Table 2 correspond to the values at small strains. While conducting a free-field analysis of the site (Subsection 3.3 ), the values adjusted to the maximum strain level in each earthquake were used [29]. Adoption of the equivalent linear approach to modeling the soil medium is considered to be adequate for the expected level of soil strains under seismic wave propagation. In the case of steel pipe, the elastic-perfectly plastic behavior was taken into account, similar to many other research works such as the one conducted by Jin et al. [30] that included steel pipe modeling.

\section{FE modeling}

\subsection{General}

The general-purpose finite element code program, ABAQUS, was used to develop the continuum model of the pipe and its surrounding soil.

To develop the buried pipe model, the pipe is discretized using the shell elements. In addition, the soil around the pipe is modeled by $3 \mathrm{D}$ solid elements following the Mohr-Coulomb behavioral rule with the assumption of a null cohesion. C3D8R elements are used for modeling the soil around the pipe. This element is a 3D solid with eight nodes and three translational degrees of freedom in each node. A surface-to-surface contact is also used for modeling friction and no-tension behavior between the pipe and soil. This contact condition allows a finite sliding and separation at the pipe-soil interface based on the Coulomb friction criterion. The friction coefficient is obtained from Eq. (1):

$$
\mu=\tan \left(\varphi_{s}\right)
$$

where $\mu$ is the friction coefficient between the pipe and

Table 1. Characteristics of the pipe.

\begin{tabular}{|c|c|c|c|c|c|c|c|}
\hline Type & $\begin{array}{l}\text { Bent radius } \\
\quad(\mathrm{mm})\end{array}$ & $\begin{array}{c}\text { Burial depth } \\
(\mathbf{m})\end{array}$ & $\begin{array}{c}\text { Mass density } \\
\left(\mathrm{kg} / \mathrm{m}^{3}\right)\end{array}$ & $\begin{array}{c}\text { Elastic modulus, } \\
E(\mathrm{GPa})\end{array}$ & $\begin{array}{l}\text { Poisson's } \\
\text { ratio }\end{array}$ & $\begin{array}{c}\text { Yield stress } \\
\sigma_{y}(\mathrm{MPa})\end{array}$ & $\begin{array}{c}\text { Ultimate stress } \\
\sigma_{u}(\mathrm{MPa})\end{array}$ \\
\hline Steel API X65 & 1200 & 1.5 & 7850 & 210 & 0.3 & 465.4 & 517.7 \\
\hline
\end{tabular}

Table 2. Characteristics of the soil types.

\begin{tabular}{ccccccc}
\hline Type & $\begin{array}{c}\text { Specific } \\
\text { weight } \\
\left(\mathbf{k N} / \mathbf{m}^{\mathbf{3}}\right)\end{array}$ & $\begin{array}{c}\text { Internal } \\
\text { friction } \\
\text { angle } \\
\boldsymbol{\varphi}(\mathbf{d e g})\end{array}$ & $\begin{array}{c}\text { Pipe-soilfriction } \\
\text { angle (deg) }\end{array}$ & $\begin{array}{c}\text { Elastic } \\
\text { modulus, } \\
\boldsymbol{E}(\mathbf{M P a})\end{array}$ & $\begin{array}{c}\text { Poisson's } \\
\text { ratio }\end{array}$ & $\begin{array}{c}\text { Average shear } \\
\text { wave velocity } \\
\boldsymbol{V}_{\mathbf{s}}(\mathbf{m} / \mathbf{s})\end{array}$ \\
\hline Loose sand & 14 & 28 & 17 & 20 & 0.3 & 75 \\
Medium sand & 18 & 35 & 21 & 40 & 0.3 & 220 \\
Dense sand & 22 & 45 & 27 & 70 & 0.3 & 450 \\
\hline
\end{tabular}




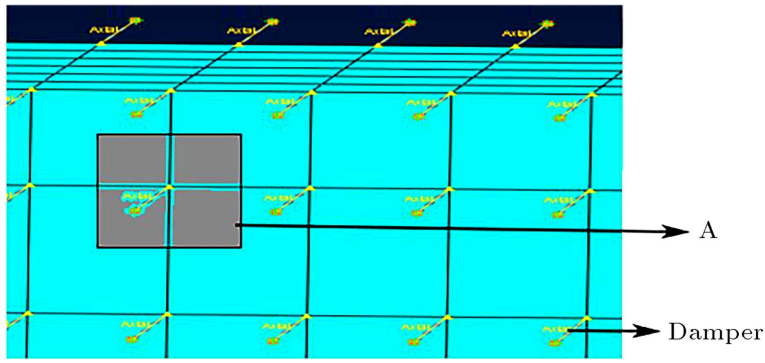

Figure 1. Arrangement of dampers on the sides of the continuum model.

soil, and $\varphi_{s}$ the pipe-soil friction angle. Dimensions of the soil model will be calculated later through sensitivity analysis.

Infinity of the soil medium was measured using dampers at the truncation planes. A set of three perpendicular dampers is employed in each node on the mentioned planes such that two of the dampers are tangent and the other one is normal to the truncation plane. Based on such arrangement, the waves propagating normal to the plane are completely absorbed and the other waves are only partially reflected [31]. Then, the dampers enable propagation of vibration energy toward the infinite volume of soil. Arrangement of dampers is shown in Figure 1. The damping coefficients of the normal dampers, $C_{n}$, and the tangential dampers, $C_{t}$, are calculated through Eq. (2) [31]:

$$
C_{n}=\rho V_{p} A, \quad C_{t}=\rho V_{s} A,
$$

where $\rho, V_{p}$, and $V_{s}$ are the density, extension wave, and shear wave velocities of soil around the pipe, respectively, and $A$ is the area shared by one finite element node.

In this study, the Rayleigh damping was used for modeling the material damping. This type of damping introduces the damping matrix as a linear combination of mass and stiffness matrices for the response modes with important contributions. The damping ratio of the pipe-soil medium is considered to be 0.04 of the critical value [26].

\subsection{Boundary conditions of the pipe}

To ensure an accurate simulation, it is necessary to consider the infinite length of the pipe away from the bend. In this study, the boundary condition proposed by Liu et al. [32] was utilized. In their study, they assumed that the lateral deformation of a pipe in far distances had only a small effect on the response of the section under study; yet, the longitudinal friction was effective.

As shown in Figure 2, the friction force, caused by the axial force $F$, along the part $\mathrm{OB}$ of the pipe contains two parts: the static friction along $\mathrm{OC}$ and the slip friction along $\mathrm{CB}$. Point $\mathrm{O}$ is fixed. A nonlinear axial spring is used at both ends of the pipe to simulate

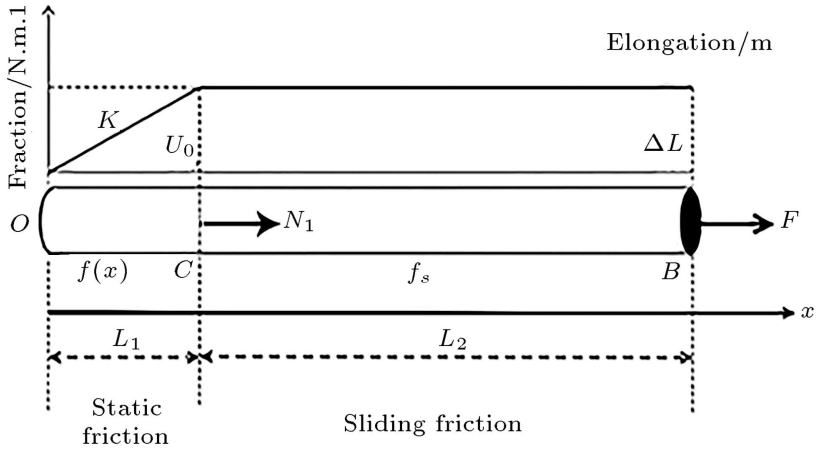

Figure 2. The boundary conditions to simulate the infinite length of the pipe [32].

this axial behavior, as shown in Figure 3. The axial force $(F)$-pipe extension $(\Delta L)$ relation at the end of the pipe can be evaluated as follows [32]:

$$
\begin{aligned}
& F(\Delta L)= \\
& \left\{\begin{array}{l}
\sqrt{\frac{3 E A f_{s}}{2}} U_{0}{ }^{-\frac{1}{6}} \Delta L^{\frac{2}{3}} \quad 0 \leq \Delta L \leq U_{0} \\
\sqrt{2 E A f_{s}\left(\Delta L-\frac{1}{4} U_{0}\right)} U_{0} \leq \Delta L \leq \frac{\sigma_{y}{ }^{2} A}{2 E f_{s}}+\frac{U_{0}}{4}
\end{array}\right.
\end{aligned}
$$

where $E$ is the elastic modulus, $A$ the section area of pipe, $f_{s}$ the slip friction force on unit length of pipe, $U_{0}$ the yield displacement, and $\sigma_{y}$ the yield stress of the pipe.

\subsection{Dynamic loading}

Earthquake magnitude, shear wave velocity of the soil on which the motion was recorded, and the epicentral or focal distance, or instead the peak ground acceleration, are effective parameters for selecting suitable ground motions. In this study, earthquakes recorded on the soils with their shear wave velocities falling in the ranges of less than $180 \mathrm{~m} / \mathrm{s}$ as loose soils, between 180 and $360 \mathrm{~m} / \mathrm{s}$ as medium soils, and between 360 and $750 \mathrm{~m} / \mathrm{s}$ as dense soils are selected separately since the dynamic analysis is done for three cases of soil types. For all of these soil types, only earthquakes with magnitudes larger than six and epicentral distances between $20-50 \mathrm{~km}$ are considered. The strong motion database PEER NGA [33] is used to select the ground motions. The earthquake records reported in Table 3 are also considered in this study. There should be at least seven earthquakes to make averaging possible between the response results. Listed in this table are all three components of different records of San Fernando (1971), Northridge (1994), Kobe (1995), ChiChi (1999), Tottori (2000), Niigata (2004), and Darfield (2010) earthquakes. The ground motion is applied to the model concurrently along the three perpendicular directions using the three components of each earthquake. The response spectra of the selected ground motions are presented in Figures 4-6 for the three soil types. The motion is input at the bottom and 

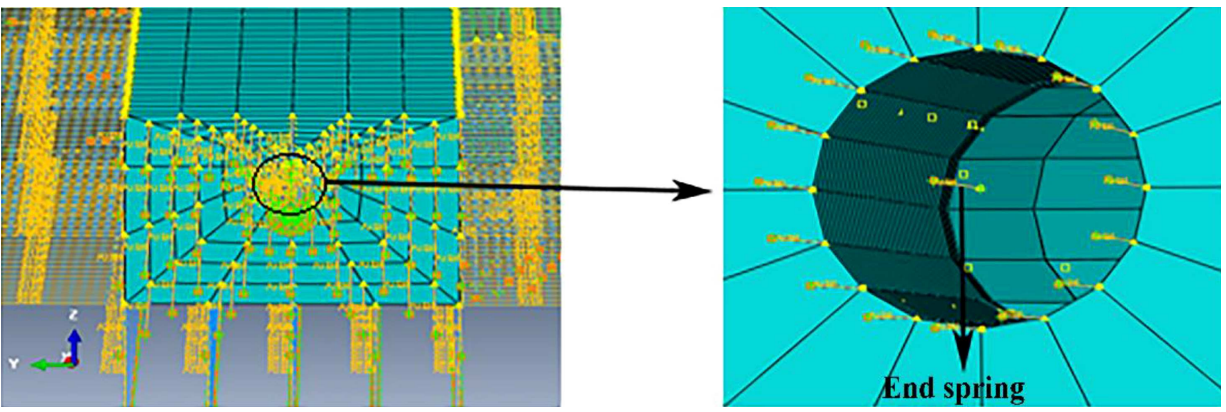

Figure 3. The end springs for simulating the infinite length of pipe.

Table 3. Characteristics of the ground motions.

\begin{tabular}{|c|c|c|c|c|c|c|}
\hline \multirow[t]{2}{*}{ Earthquake } & Station & $\begin{array}{c}\text { Shear wave } \\
\text { velocity, } V_{s}(\mathrm{~m} / \mathrm{s})\end{array}$ & \multicolumn{3}{|c|}{$\begin{array}{c}\text { Peak ground } \\
\text { acceleration }(\mathrm{g}) \\
x \text {-direction, } y \text {-direction, } \\
z \text {-direction }\end{array}$} & \multirow{2}{*}{ 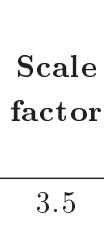 } \\
\hline & Whittier Narrows Dam & $V_{s}<180$ & 0.108 & 0.101 & 0.033 & \\
\hline \multirow[t]{2}{*}{ San Fernando } & Santa Felita Dam & $180<V_{s}<360$ & 0.154 & 0.154 & 0.067 & 1.3 \\
\hline & Lake Hughes \# 12 & $360<V_{s}<750$ & 0.382 & 0.282 & 0.194 & 0.7 \\
\hline \multirow{3}{*}{ Northridge } & Montebello-Bluff Rd & $V_{s}<180$ & 0.179 & 0.129 & 0.076 & 2.3 \\
\hline & LA-Centinela St & $180<V_{s}<360$ & 0.465 & 0.322 & 0.109 & 0.85 \\
\hline & Santa Monica City Hall & $360<V_{s}<750$ & 0.883 & 0.37 & 0.23 & 0.55 \\
\hline \multirow{3}{*}{ Kobe } & OSAJ & $V_{s}<180$ & 0.082 & 0.067 & 0.065 & 5.3 \\
\hline & Fukushima & $180<V_{s}<360$ & 0.184 & 0.215 & 0.198 & 1.7 \\
\hline & Nishi-Akashi & $360<V_{s}<750$ & 0.483 & 0.464 & 0.386 & 0.55 \\
\hline \multirow{3}{*}{ ChiChi } & CHY041 & $V_{s}<180$ & 0.639 & 0.302 & 0.123 & 0.85 \\
\hline & CHY028 & $180<V_{s}<360$ & 0.821 & 0.653 & 0.337 & 0.5 \\
\hline & CHY080 & $360<V_{s}<750$ & 0.902 & 0.968 & 0.724 & 0.45 \\
\hline \multirow{3}{*}{ Tottori } & SMN002 & $V s<180$ & 0.179 & 0.153 & 0.107 & 2.75 \\
\hline & SMN001 & $180<V_{s}<360$ & 0.251 & 0.235 & 0.087 & 1 \\
\hline & OKY004 & $360<V_{s}<750$ & 0.824 & 0.538 & 0.172 & 0.35 \\
\hline \multirow{3}{*}{ Darfield } & DORC & $V_{s}<180$ & 0.086 & 0.082 & 0.076 & 4.25 \\
\hline & Canterbury Aero Club & $180<V_{s}<360$ & 0.198 & 0.185 & 0.300 & 1.3 \\
\hline & DFHS & $360<V_{s}<750$ & 0.512 & 0.471 & 0.373 & 0.7 \\
\hline \multirow{3}{*}{ Niigata } & NIG014 & $V_{s}<180$ & 0.12 & 0.098 & 0.077 & 3.2 \\
\hline & NIG017 & $180<V_{s}<360$ & 0.476 & 0.378 & 0.333 & 0.65 \\
\hline & NIGH01 & $360<V_{s}<750$ & 0.837 & 0.669 & 0.379 & 0.4 \\
\hline
\end{tabular}

side nodes with a node-to-node delay corresponding to the wave velocity and its angle of incidence. The SHAKE2000 program is used for deconvolution of the ground motion recorded on the surface to the motion at depth in free-field analysis [34]. In this program, the mechanical characteristics of soils at large strains are used based on maximum strain level in each earthquake [29].
To ensure the consistency of the results, it is necessary to scale the earthquake records. Consistency of results implies that the responses belong to earthquakes with more or less the same return periods to make averaging meaningful. The process of scaling makes the response spectra of individual earthquakes "similar" to each other. To this end, Square Root of the Sum of the Squares (SRSS) response spectrum is calculated 


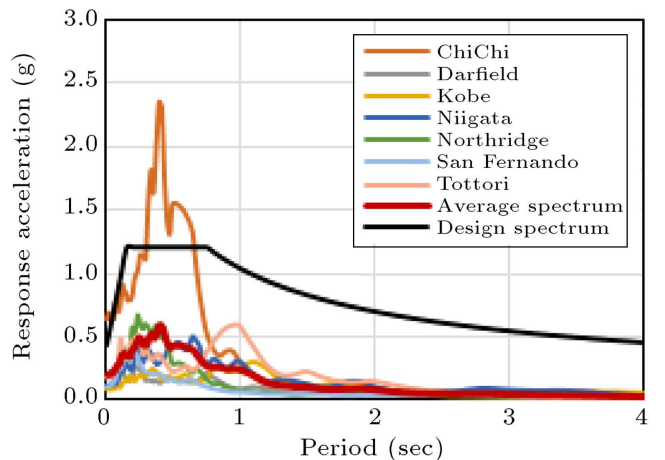

(a)

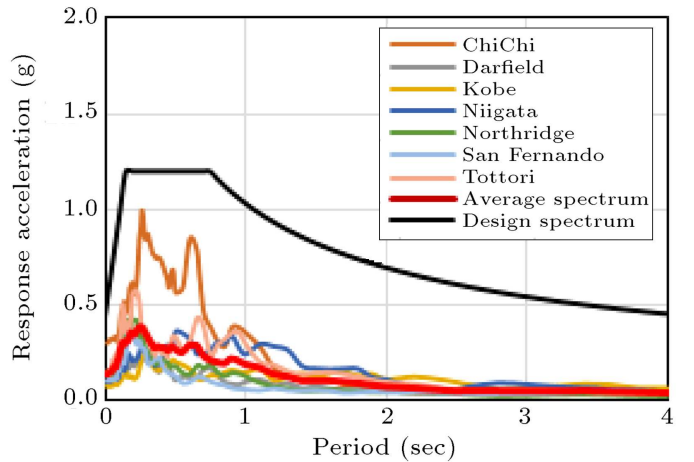

(b)

Figure 4. Response spectra of earthquakes for loose sand: (a) $x$-direction and (b) $y$-direction.

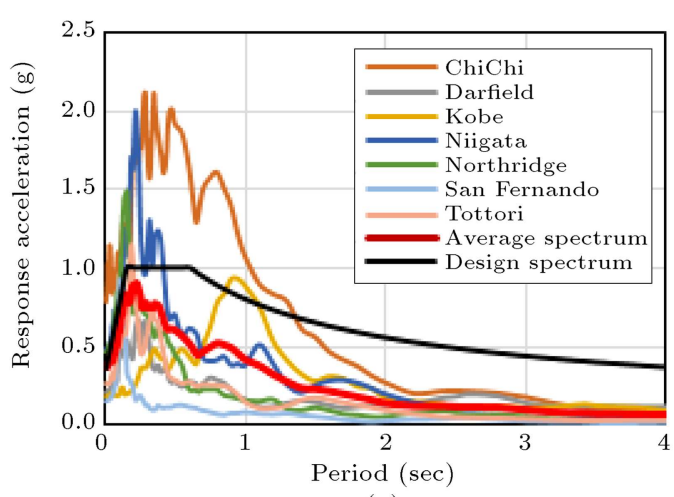

(a)

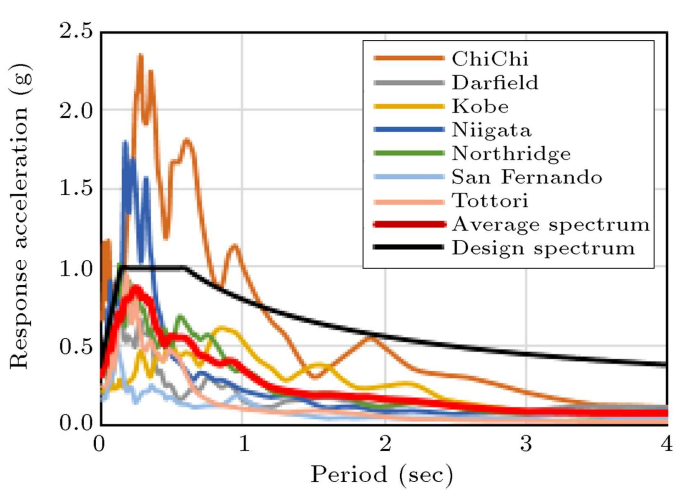

(b)

Figure 5. Response spectra of earthquakes for medium sand: (a) $x$-direction and (b) $y$-direction.

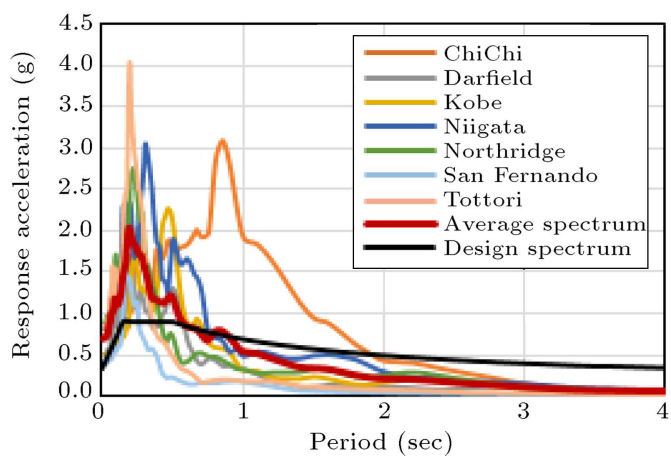

(a)

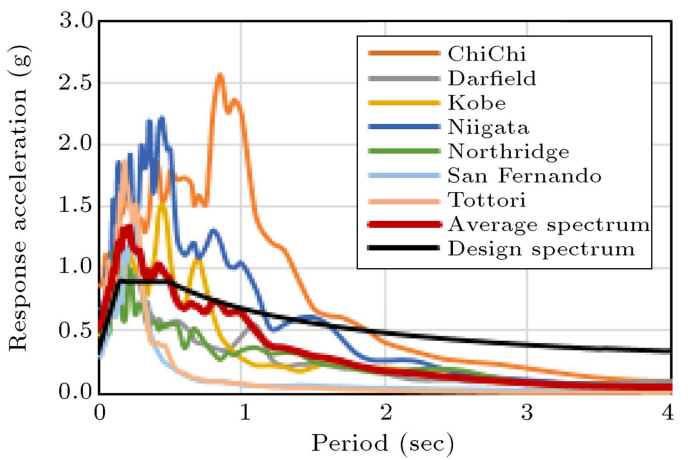

(b)

Figure 6. Response spectra of earthquakes for dense sand: (a) $x$-direction and (b) $y$-direction.

for the perpendicular horizontal components of each earthquake. The average of these spectra is scaled such that it does not fall below the design spectrum in the period range of $0.2 T$ to $1.5 T$ where $T$ is the fundamental period of the pipe-soil system [35]. The design spectra are also shown in Figures 4-6. They belong to a highly seismic area with the soil types mentioned in this study [35]. The fundamental periods of the three pipe-soil systems are proved to be 0.53 , 0.42 , and $0.35 \mathrm{sec}$ for the loose, medium, and dense sands, respectively, and the scale factors are presented in Table 3 for each earthquake.
One of the key parameters in the dynamic pipe response is the incidence angle of the coming waves. In this study, the vertical and horizontal propagation of seismic waves is considered separately. In vertical propagation, the input motion is synchronized in the input motion nodes. In horizontal propagation, the propagation direction is assumed to be parallel to a pipe branch ( $x$-direction). Then, the input motion is applied to the nodes at one end of the pipe, and the same motion is applied to the other nodes in the path of propagation with a time delay proportional to distance from the end nodes and wave velocity. 
Table 4. Characteristics of the analysis cases $(\theta=$ elbow angle).

\begin{tabular}{|c|c|c|c|c|c|c|c|c|c|c|c|}
\hline \multirow{2}{*}{$\begin{array}{l}\text { Model } \\
\text { number }\end{array}$} & \multirow[b]{2}{*}{$\theta(\operatorname{deg})$} & \multirow[b]{2}{*}{$V_{s}(\mathrm{~m} / \mathrm{s})$} & \multirow[b]{2}{*}{$D / t$} & \multicolumn{8}{|c|}{ Maximum PGA (g) between different components } \\
\hline & & & & San & Fernando & Northridge & Kobe & ChiChi & Tottori & Darfield & Niigata \\
\hline 1 & 90 & 450 & 21 & & 0.382 & 0.883 & 0.483 & 0.968 & 0.824 & 0.512 & 0.837 \\
\hline 2 & 90 & 450 & 42 & & 0.382 & 0.883 & 0.483 & 0.968 & 0.824 & 0.512 & 0.837 \\
\hline 3 & 90 & 450 & 63 & & 0.382 & 0.883 & 0.483 & 0.968 & 0.824 & 0.512 & 0.837 \\
\hline 4 & 90 & 450 & 84 & & 0.382 & 0.883 & 0.483 & 0.968 & 0.824 & 0.512 & 0.837 \\
\hline 5 & 90 & 220 & 21 & & 0.154 & 0.465 & 0.215 & 0.821 & 0.251 & 0.3 & 0.476 \\
\hline 6 & 90 & 220 & 42 & & 0.154 & 0.465 & 0.215 & 0.821 & 0.251 & 0.3 & 0.476 \\
\hline 7 & 90 & 220 & 63 & & 0.154 & 0.465 & 0.215 & 0.821 & 0.251 & 0.3 & 0.476 \\
\hline 8 & 90 & 220 & 84 & & 0.154 & 0.465 & 0.215 & 0.821 & 0.251 & 0.3 & 0.476 \\
\hline 9 & 90 & 75 & 21 & & 0.108 & 0.179 & 0.082 & 0.639 & 0.179 & 0.086 & 0.12 \\
\hline 10 & 90 & 75 & 42 & & 0.108 & 0.179 & 0.082 & 0.639 & 0.179 & 0.086 & 0.12 \\
\hline 11 & 90 & 75 & 63 & & 0.108 & 0.179 & 0.082 & 0.639 & 0.179 & 0.086 & 0.12 \\
\hline 12 & 90 & 75 & 84 & & 0.108 & 0.179 & 0.082 & 0.639 & 0.179 & 0.086 & 0.12 \\
\hline 13 & 112.5 & 450 & 42 & & 0.382 & 0.883 & 0.483 & 0.968 & 0.824 & 0.512 & 0.837 \\
\hline 14 & 112.5 & 220 & 42 & & 0.154 & 0.465 & 0.215 & 0.821 & 0.251 & 0.3 & 0.476 \\
\hline 15 & 112.5 & 75 & 42 & & 0.108 & 0.179 & 0.082 & 0.639 & 0.179 & 0.086 & 0.12 \\
\hline 16 & 135 & 450 & 42 & & 0.382 & 0.883 & 0.483 & 0.968 & 0.824 & 0.512 & 0.837 \\
\hline 17 & 135 & 220 & 42 & & 0.154 & 0.465 & 0.215 & 0.821 & 0.251 & 0.3 & 0.476 \\
\hline 18 & 135 & 75 & 42 & & 0.108 & 0.179 & 0.082 & 0.639 & 0.179 & 0.086 & 0.12 \\
\hline 19 & 157.5 & 450 & 42 & & 0.382 & 0.883 & 0.483 & 0.968 & 0.824 & 0.512 & 0.837 \\
\hline 20 & 157.5 & 220 & 42 & & 0.154 & 0.465 & 0.215 & 0.821 & 0.251 & 0.3 & 0.476 \\
\hline 21 & 157.5 & 75 & 42 & & 0.108 & 0.179 & 0.082 & 0.639 & 0.179 & 0.086 & 0.12 \\
\hline 22 & 180 & 450 & 42 & & 0.382 & 0.883 & 0.483 & 0.968 & 0.824 & 0.512 & 0.837 \\
\hline 23 & 180 & 220 & 42 & & 0.154 & 0.465 & 0.215 & 0.821 & 0.251 & 0.3 & 0.476 \\
\hline 24 & 180 & 75 & 42 & & 0.108 & 0.179 & 0.082 & 0.639 & 0.179 & 0.086 & 0.12 \\
\hline
\end{tabular}

\section{Numerical results}

\subsection{General}

In the following parts, the values of the maximum pipe axial strain of the bend are presented versus the incidence angle of seismic waves, bend angle, pipe diameter, pipe wall thickness, and soil type. In this regard, the tensile and compressive strains are normalized to the yield strain $\left(\varepsilon_{y}\right)$ and critical strain $\left(\varepsilon_{c r}\right)$, respectively. The yield strain is determined by Hook's law to be 0.002 according to the characteristics given in Table 1 . The critical strain is calculated using the equation proposed by the American Lifelines Alliance [36], appearing as in Eq. (4) below:

$$
\begin{aligned}
\varepsilon_{c r} & =0.75\left[0.5 \frac{t}{D^{\prime}}-0.0025+3000\left(\frac{p D}{2 E t}\right)^{2}\right], \\
D^{\prime} & =\frac{D}{1-\frac{3}{D}\left(D-D_{\min }\right)}
\end{aligned}
$$

where $E$ is the elastic modulus, $t$ the thickness, $D$ the diameter equal to $400 \mathrm{~mm}, p$ the internal pressure of the pipe assumed to be negligible in this study, and
$D_{\min }$ the inner diameter of the pipe. For the $D / t$ cases of this study, $t$ appears to be in the range of 5 to $20 \mathrm{~mm}$.

As shown in Table 4, 24 different cases, each one under seven earthquakes, are considered in the analysis of this study.

\subsection{Sensitivity analysis on model dimensions}

To determine the optimal model dimensions, the length of the straight part of the pipe on each side of the bend is considered to be in the range of $45 D$ to $180 D$ ( $D=$ pipe diameter) in different cases, and the maximum axial strain is calculated at the bend. A sample of results is presented in Figures 7 and 8 for a pipe under vertical propagation of the Northridge earthquake. As shown in Figure 7, a $90 D$ straight segment on each side of the bend is enough for response calculation at the bend. As observed in Figure 8, the containing part of the soil that perpendicularly extends a distance equal to $10 D$ from the center of pipe to each side leads to a response with good accuracy. Based on the analysis similar to what is presented in Figure 8, the depth of the model below the pipe is also considered to be $10 D$. Similarly, several configurations are taken into account in the arrangement of the finite elements. In the longitudinal direction, a regular setting of elements of 


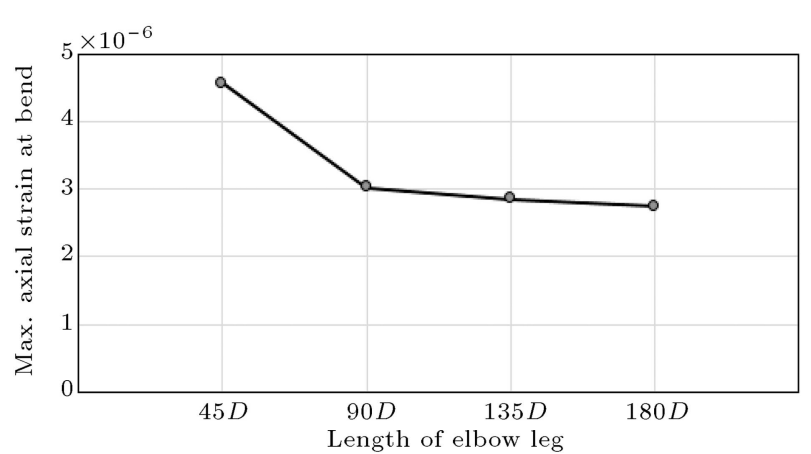

Figure 7. Sensitivity analysis for the straight lengths of the pipe $\left(D=40 \mathrm{~cm}, t=9.5 \mathrm{~mm}, \theta=180^{\circ}\right.$, vertical propagation).

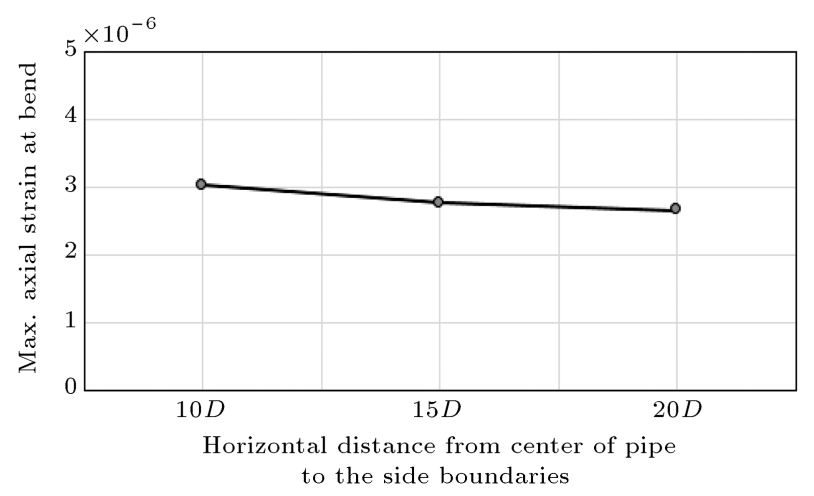

Figure 8. Sensitivity analysis for the model dimension ( $D=40 \mathrm{~cm}, t=9.5 \mathrm{~mm}, \theta=180^{\circ}$, vertical propagation).

the same size, each one being $3 D$ in its dimension along the pipe length, is proved to be efficient and accurate enough to display variations in the strains in the same direction. At the cross-section, a circumferential arrangement at a constant central angle of $22.5^{\circ}$ and a radial arrangement dividing the distance to the related boundary to 7 equal parts are proved to be the most efficient one. Selecting a finer mesh does not add to accuracy, while it lengthens the computational time with no benefit. Therefore, the model dimensions are given in Figure 9. Of note, introducing appropriate boundary conditions is quite decisive when determining

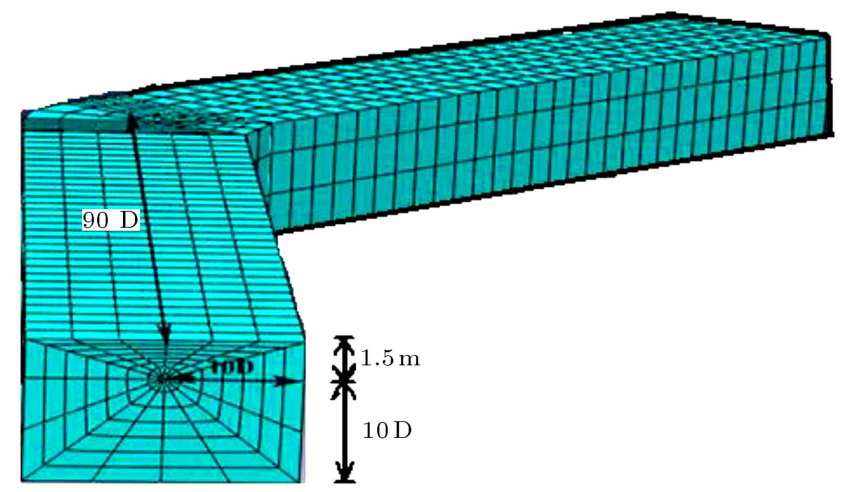

Figure 9. Geometrical dimensions and the finite-element discretization of the system.

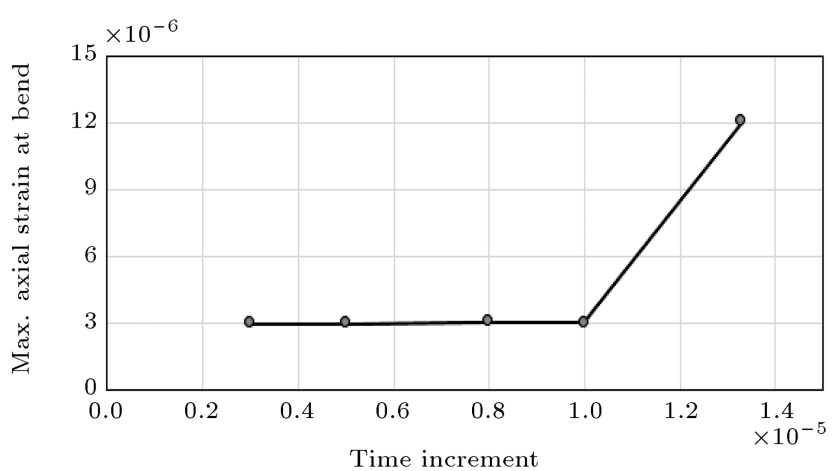

Figure 10. Sensitivity analysis for the time increment ( $D=40 \mathrm{~cm}, t=9.5 \mathrm{~mm}, \theta=180^{\circ}$, vertical propagation).

the model dimensions in its plan and elevation. The dimensions of the model that are relatively small in its elevation directly result from placing normal dampers at the boundary nodes.

\subsection{Sensitivity analysis on the time increment} Dynamic analysis of the current system is performed using explicit time integration mainly because only a diagonal mass matrix is used to solve the momentary equations of unknown nodal displacements in this method. However, care should be taken when selecting the time increment for convergency purposes in such a procedure. This study also employs the explicit nonlinear dynamic analysis. Due to the complexity and large size of the model, special attention should be dedicated to minimizing the run time alongside keeping enough accuracy. This aspect pertains to an optimal time step selection. Pipe response under vertical propagation of the Northridge earthquake is shown in Figure 10. As observed, at time increments not larger than $1.0 \mathrm{e}-5 \mathrm{sec}$, the analysis results are almost insensitive to the size of the time step. This is the selected time increment for the strain analysis of this study.

\subsection{Analysis under vertical propagation}

The analysis results for maximum axial strain at the bend when the wave propagation direction is assumed to be vertical are presented in Figure 11 at different elbow angles. The positive and negative values indicate the tensile and compressive strains, respectively. As observed, the strain values are very small and are generally around zero for vertical propagation. Since the seismic motion is uniformly input at the base nodes under vertical propagation, the inertial effects are prevailing in the response. Therefore, the response acceleration is regarded as the primary cause of the strains. As seen in Figures 4-6, in the stiffer soils, the acceleration spectra possess larger values on average in the range of the natural periods of the site under study. Based on the above facts, it can be anticipated that the maximum pipe strain values are larger for the stiffer soils under vertical propagation. Of course, this 


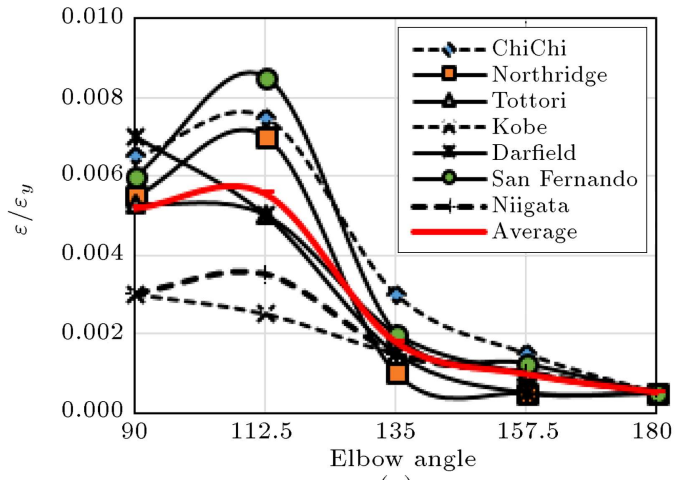

(a)

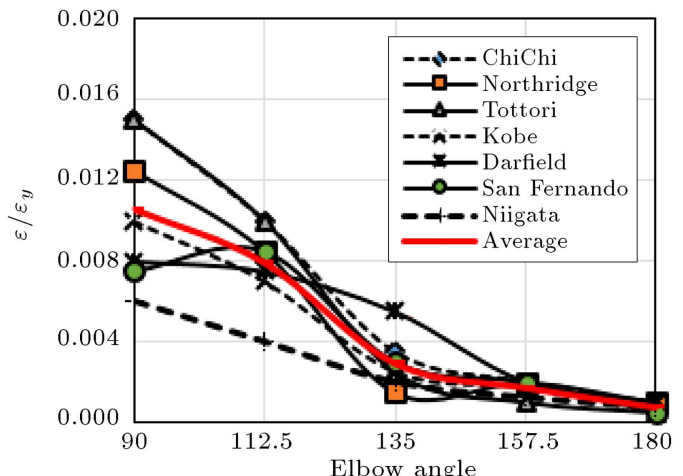

(c)

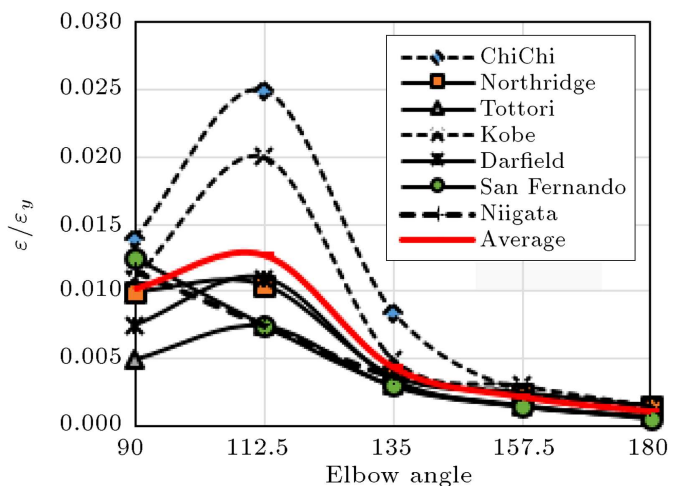

(e)

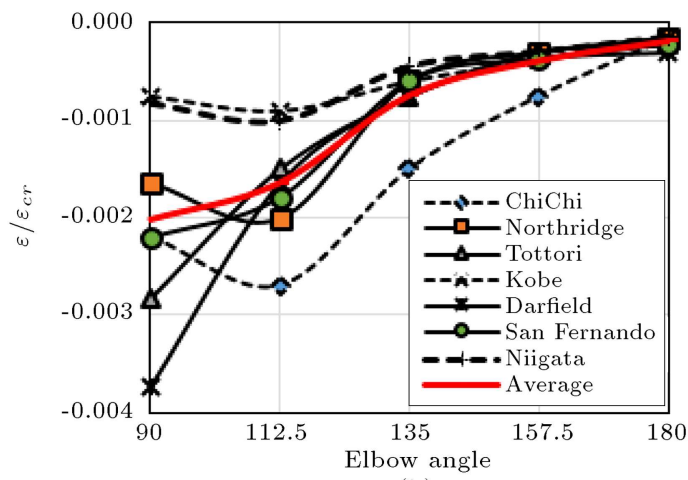

(b)

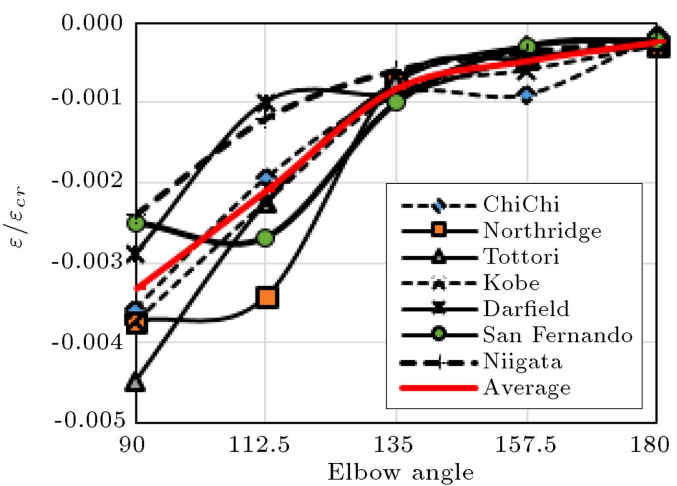

(d)

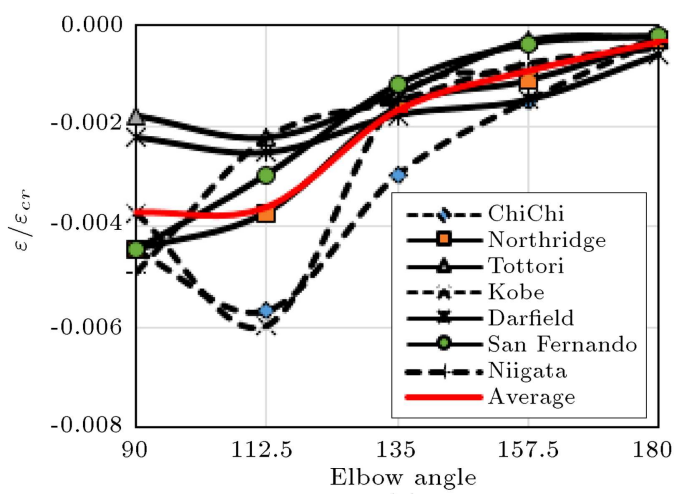

(f)

Figure 11. Maximum axial strain at a bend against elbow angle for vertical propagation $(D=40 \mathrm{~cm}, t=9.5 \mathrm{~mm}):(\mathrm{a})$ Tensile strain, loose sand, (b) compressive strain, loose sand, (c) tensile strain, medium sand, (d) compressive strain, medium sand, (e) tensile strain, dense sand, and (f) compressive strain, dense sand.

is the case as the maximum response almost doubles from softer to stiffer soils in Figure 11. This is in line with the findings of similar previous studies such as that conducted by Saberi et al. [26]. Both of the compressive and tensile strains reach their maximum values for the elbow angle range of $90-112.5^{\circ}$.

\subsection{Analyzing for horizontal propagation}

Figure 12 presents the analysis results of the horizontal wave propagation at different elbow angles. According to this figure, the strain values for horizontal propagation are generally much larger than the vertical propagation due to the added effect of kinematic (nonuniform) response. In addition, the strains are larger in stiffer soils. As stated in the previous sections, the length of each pipe branch in the model is $36 \mathrm{~m}$ and the shear wave velocities in the loose, medium, and stiff soils are 75, 220, and $450 \mathrm{~m} / \mathrm{sec}$, respectively. This results in the periods of loading of $0.5,0.2$, and $0.1 \mathrm{sec}$ for the same order of soils. Since the fundamental periods of the same sites are about $0.5,0.4$, and $0.35 \mathrm{sec}$, it can be concluded that the inertial effects (due to resonance) for the soft soil and the kinematic response (due to nonuniform soil movement) for the stiffer soils are prevailing. To be specific, in the case of the straight pipe in the soft 


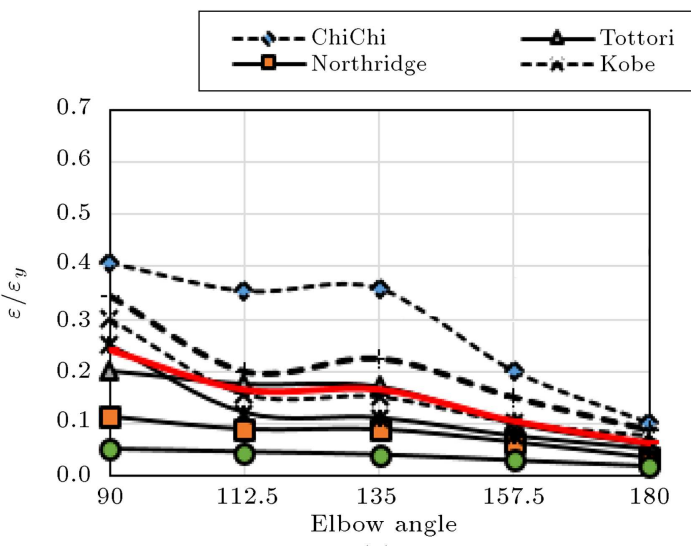

(a)

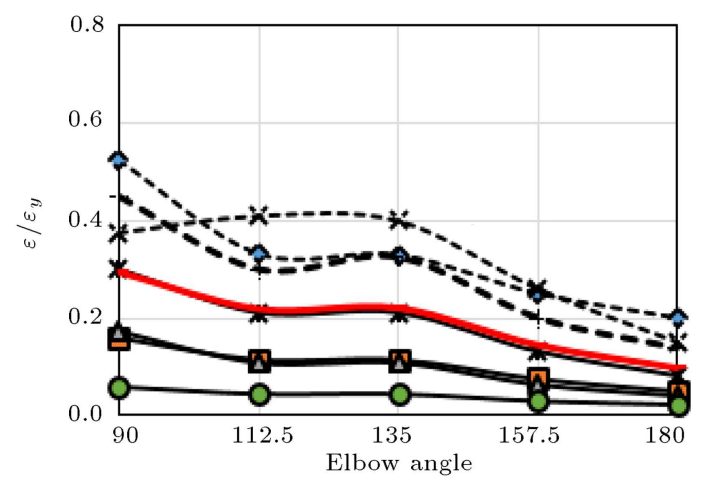

(c)

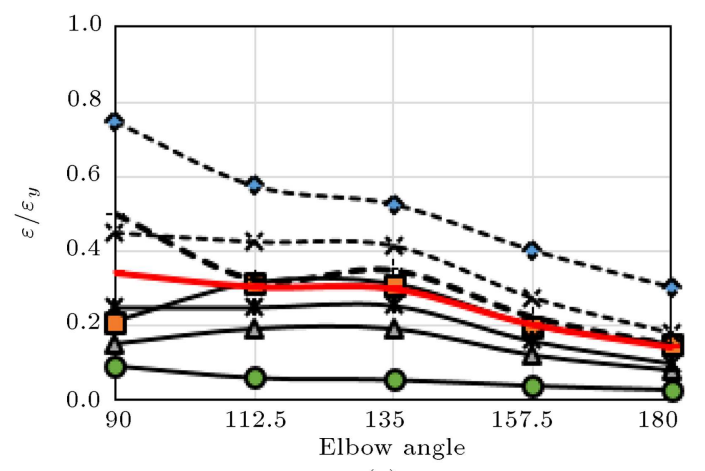

(e)

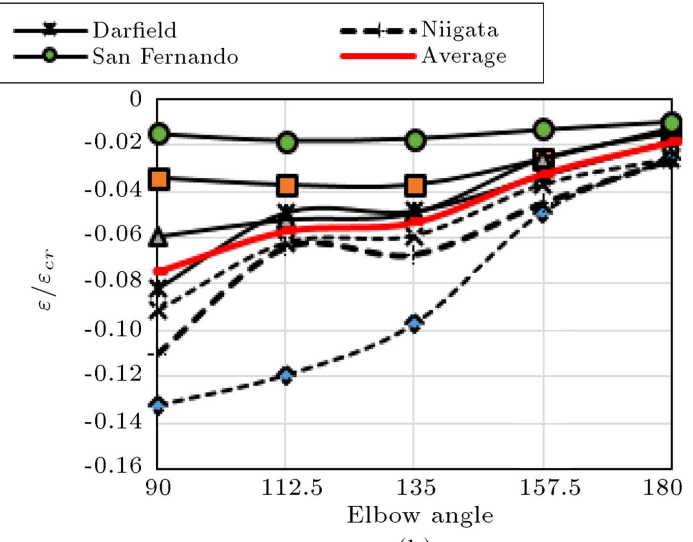

(b)

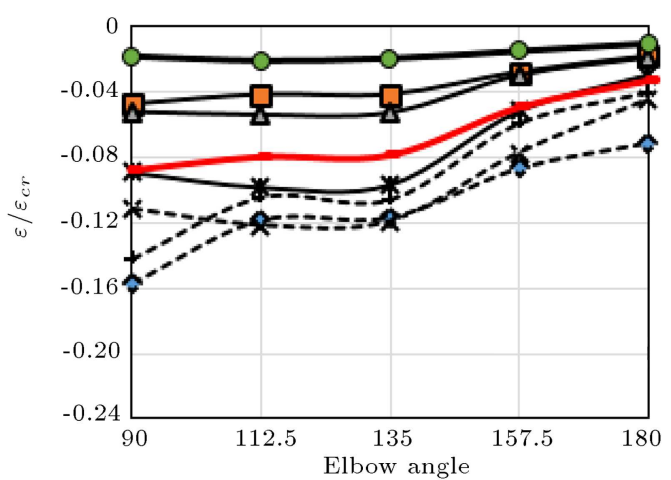

(d)

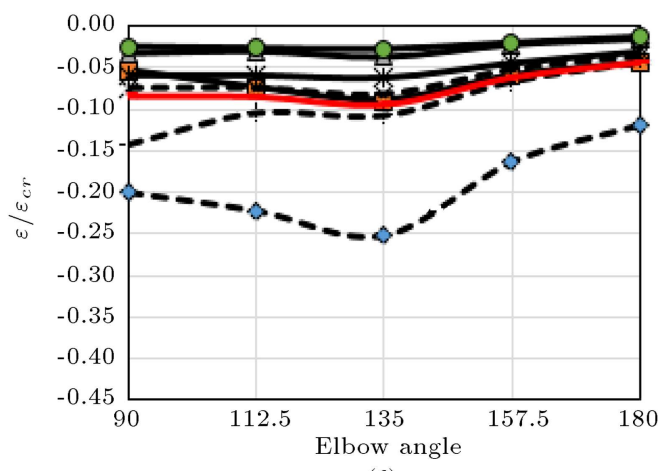

(f)

Figure 12. Maximum axial strain at a bend against elbow angle for horizontal propagation $(D=40 \mathrm{~cm}, t=9.5 \mathrm{~mm}):(\mathrm{a})$ Tensile strain, loose sand, (b) compressive strain, loose sand, (c) tensile strain, medium sand, (d) compressive strain, medium sand, (e) tensile strain, dense sand and (f) compressive strain, dense sand.

soil, the governing inertial response forces the pipe to move through the soil because of its small mass with regard to the soil. On the other hand, in the case of the same pipe in the stiffer soils, the pipe tends to have a differential movement along its length due to the governing kinematic response, thus having larger strains than those for soft soil. A similar trend was also reported in previous studies $[22,26]$.

As shown in Figures 11 and 12, the axial strain has the largest values in almost $60 \%, 30 \%$, and $10 \%$ of the cases for the bend angles of $90,112.5$, and $135^{\circ}$, respectively. This result is partially in agreement with those from the study of Ogawa and Koike [22] who concluded that the 90-degree bend angle pertained to higher critical values for pipeline strains. However, the maximum response for the elbow angle range of 90$135^{\circ}$ did not considerably vary.

\subsection{Effect of $D / t$}

Because of the axial strains being much larger with the horizontal propagation of waves and a $90^{\circ}$ bend angle, effect of $D / t$ has been also investigated for the same cases. The maximum axial strain for different ratios of pipe diameter to thickness $(D / t)$ at the bend with the angle of $90^{\circ}$ and horizontal wave propagation is presented in Figure 13. 


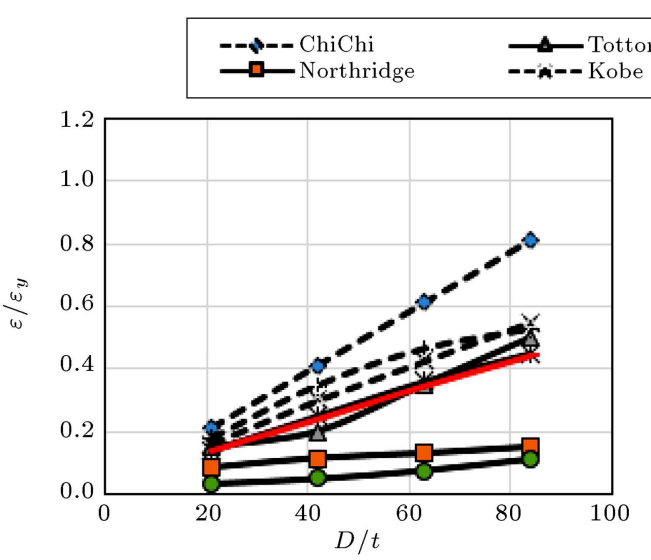

(a)

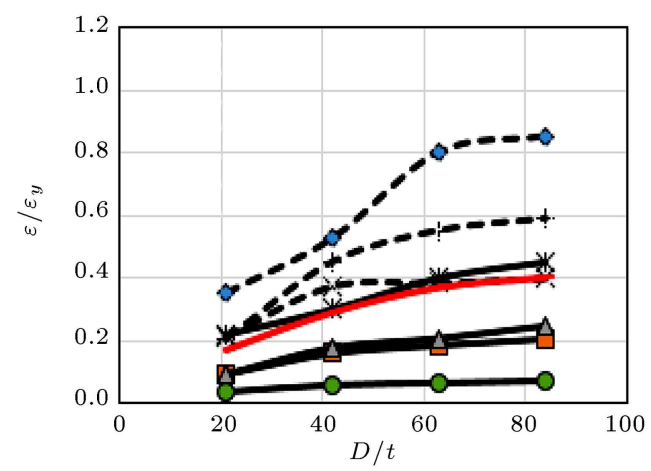

(c)

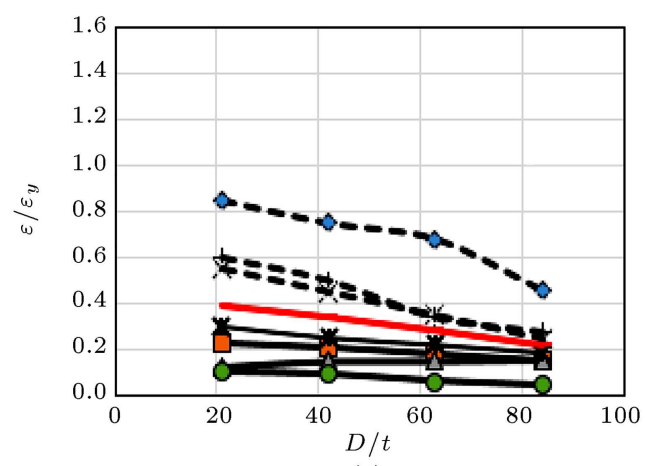

(e)

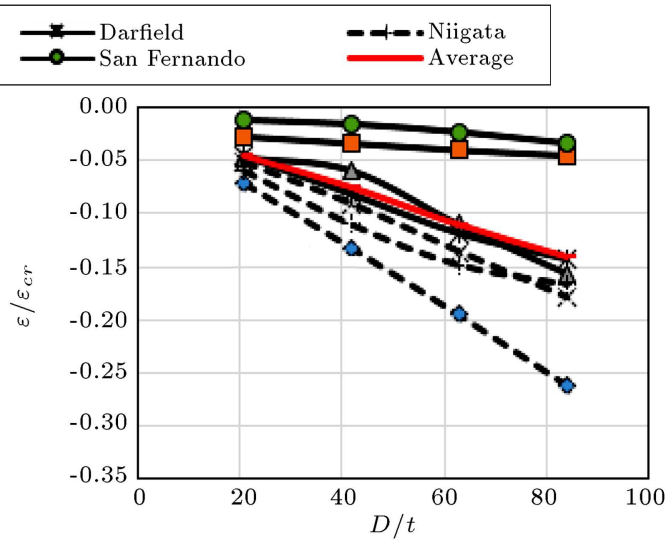

(b)

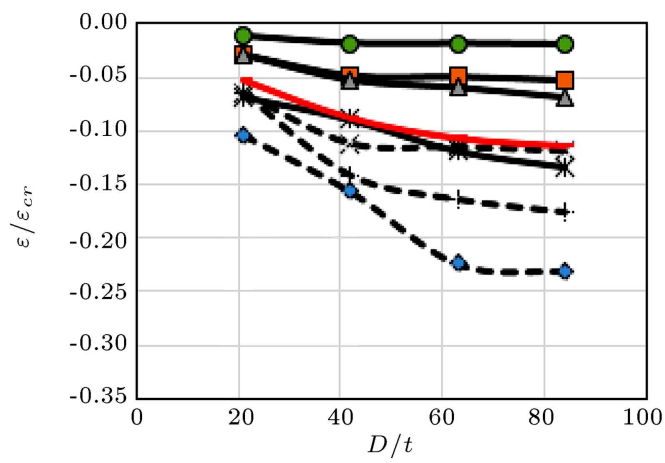

(d)

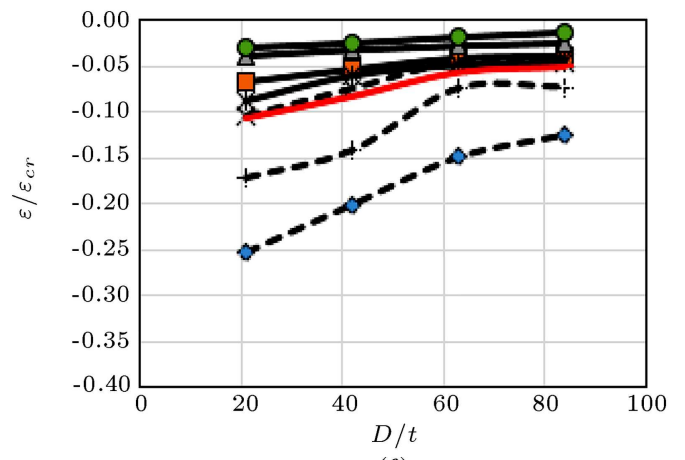

(f)

Figure 13. Effect of $D / t$ on the maximum axial strain at a bend $\left(\theta=90^{\circ}\right.$, horizontal propagation): (a) Tensile strain, loose sand, (b) compressive strain, loose sand, (c) tensile strain, medium sand, (d) compressive strain, medium sand, (e) tensile strain, dense sand, and (f) compressive strain, dense sand.

In the case of the softer soil, $D / t$ affects the response more extensively. In the loose sand, the axial strain value is larger for a more flexible pipe (larger $D / t)$. In the medium sand, the trend is similar to the loose sand, but the response is less sensitive to the variation of $D / t$. In the dense sand, the axial strain decreases for more flexible pipes. It is interesting to note that the pipe stiffness overcomes the smaller soil stiffness values in loose and medium sands; therefore, for stiffer pipes (smaller values of $D / t$ ), the axial strain decreases. On the contrary, in the dense sand effect of soil stiffness governs and for softer pipes (larger values of $D / t$ ), the axial strain decreases. By comparing the average curves in different sands, it is observed that for a $D / t$ equal to 50 , an almost identical maximum strain occurs in different sands. It is hinting that in such a case the opposite effects of the pipe stiffness and the soil stiffness cancel each other. As a result, upon increasing the soil stiffness, the pipe strain increases and decreases for $D / t<50$ and $D / t>50$, respectively.

\subsection{Comparison of the continuum model with the beam and beam-shell models}

To analyze a buried pipe, different modeling methods including continuum, beam, and beam-shell hybrid models were employed. The continuum model was used in the previous sections for calculating the maximum axial strain. The strain values of this study were com- 


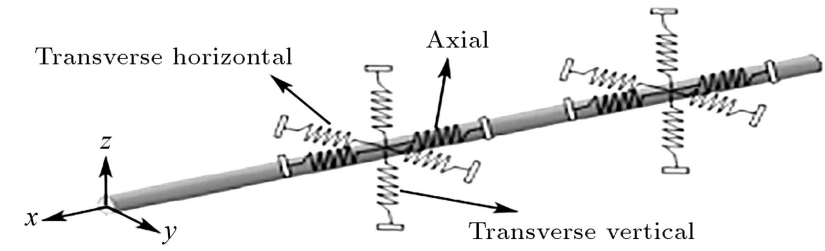

Figure 14. Arrangement of springs in the beam model.

pared with those of the study conducted by Saberi et al. [26]. They studied the beam and beam-shell hybrid models under Northridge and ChiChi earthquakes.

In the beam model, both of the pipe and soil around it were modeled using the beam element and a number of nonlinear springs, respectively, as shown in Figure 14. In this model, only estimation of the axial and bending deformations is possible.

The springs are active only under compression. The specifications of the springs can be found in American Lifeline Alliance [36]. To make a comparison of the continuum model, the stiffness properties of the soil springs were taken from Saberi et al. [26], as shown in Table 5.

In the beam-shell hybrid model, the bend and part of the straight zone of the pipe extending $15 \mathrm{D}$ away from the center of bend were modeled by shell elements. Beam elements were used for modeling the rest of the pipe [26]. The arrangement of the springs around the shell part of the hybrid model is depicted in Figure 15.

Figure 16 presents a comparison between the maximum axial bend strains calculated by the continuum model in this study and those by the beam and beam-shell models in the study of Saberi et al. [26]. The results are shown for a pipe with a $90^{\circ}$ bend and horizontal propagation of waves under the Northridge and ChiChi earthquakes. As shown, the results are in good agreement only in the general trend, not in values.

Modeling and analysis of a pipe with beam elements is simply done within a short time span; however,

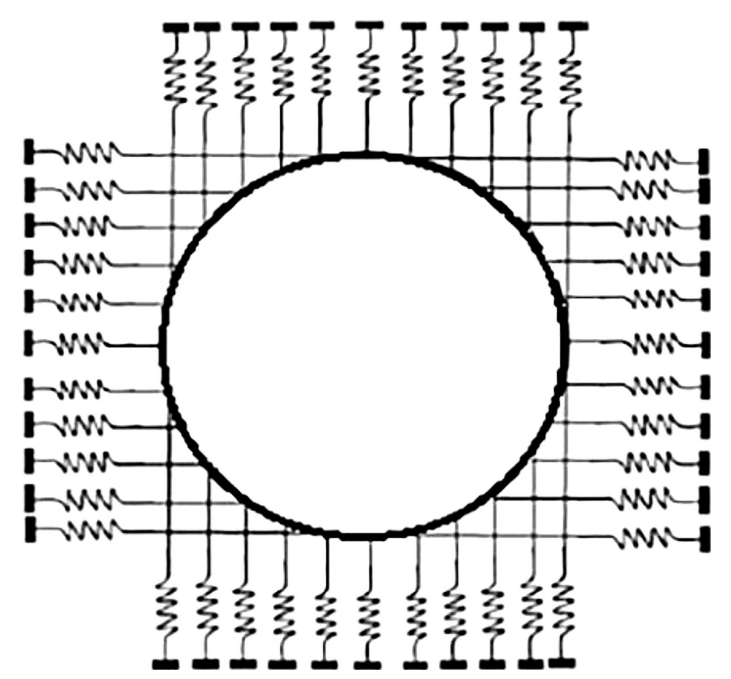

Figure 15. Arrangement of springs around the shell part of the hybrid model.

the accuracy of the results is obviously lower than those of the shell and continuum models. Moreover, the hoop stresses and strains and large deformations cannot be directly calculated employing the beam model. On the contrary, the continuum model is the most rigorous (and time consuming) model among the three approaches with the highest accuracy.

Figure 17 presents a comparison of the maximum axial bend strains calculated using the three mentioned models. A pipe with a $90^{\circ}$ bend was studied under horizontal propagation of waves to determine the maximum responses averaged between earthquakes. In addition, the principal normal strains at the bend for the three models were compared, as shown in Figure 18. Upon comparing the values of the axial strains, it was clearly observed that the beam and hybrid models considerably underestimated the response. The difference lies in the order of some $30 \%$ compared with the continuum model. Surprisingly, very good agreement is observed among the three models while estimating the principal

Table 5. The principal strain angle $(\mathrm{deg})(D=40 \mathrm{~cm}$ and $t=9.5 \mathrm{~mm})$.

\begin{tabular}{lccc}
\hline & \multicolumn{3}{c}{ Soil type } \\
\cline { 2 - 4 } Earthquake & Loose sand & Medium sand & Dense sand \\
\hline San Fernando & 38.06 & 40.93 & 39.05 \\
Northridge & 39.96 & 37.53 & 40.27 \\
Kobe & 38.53 & 40.93 & 40.01 \\
ChiChi & 37.04 & 38.07 & 39.07 \\
Tottori & 38.95 & 37.98 & 43.93 \\
Darfield & 37.03 & 36.8 & 37.45 \\
Niigata & 37.07 & 38.33 & 39.18 \\
Average & 38.02 & 38.54 & 39.58 \\
\hline
\end{tabular}




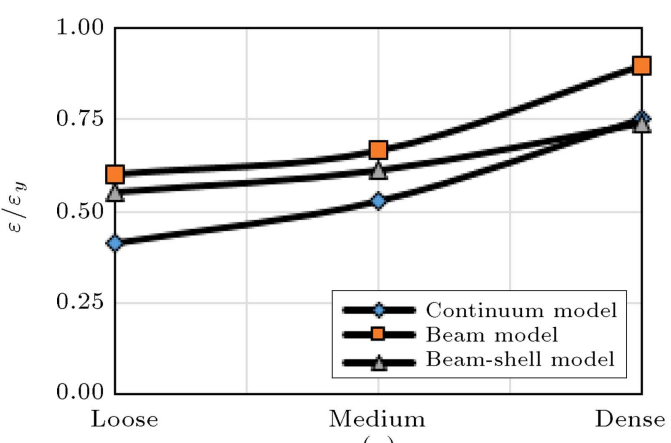

(a)

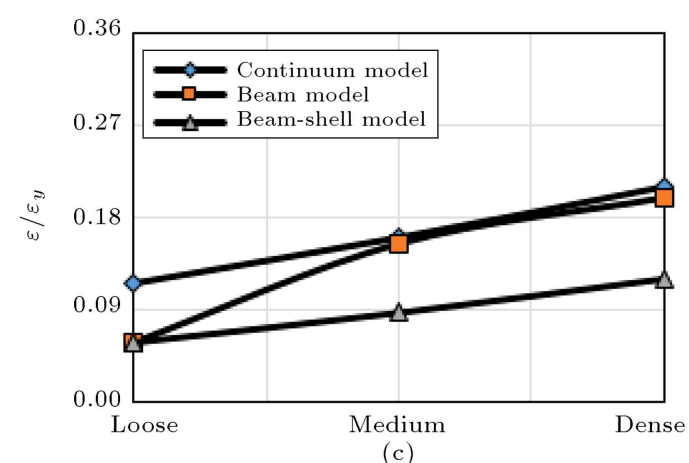

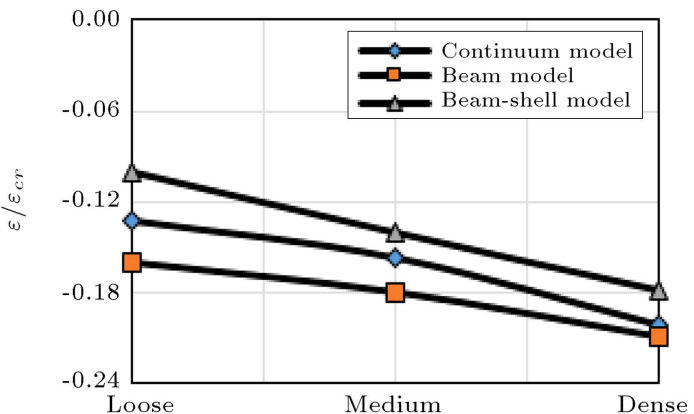

(b)

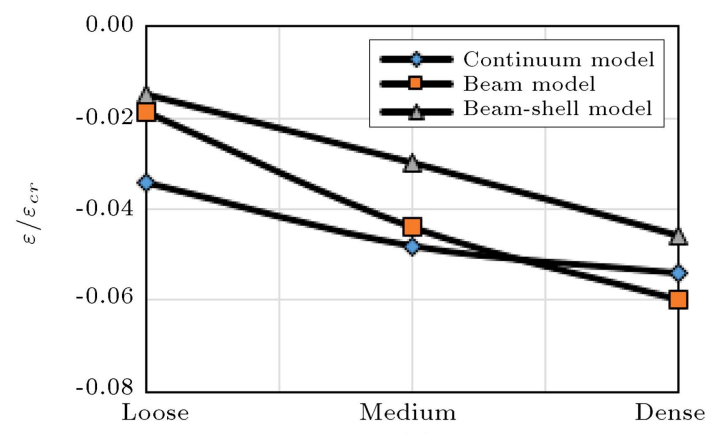

(d)

Figure 16. Maximum axial strain at a bend calculated by different methods $\left(D=40 \mathrm{~cm}, t=9.5 \mathrm{~mm}, \theta=90^{\circ}\right.$, horizontal propagation): (a) Tensile strain, ChiChi earthquake, (b) compressive strain, ChiChi earthquake, (c) tensile strain, Northridge earthquake, and (d) compressive strain, Northridge earthquake.

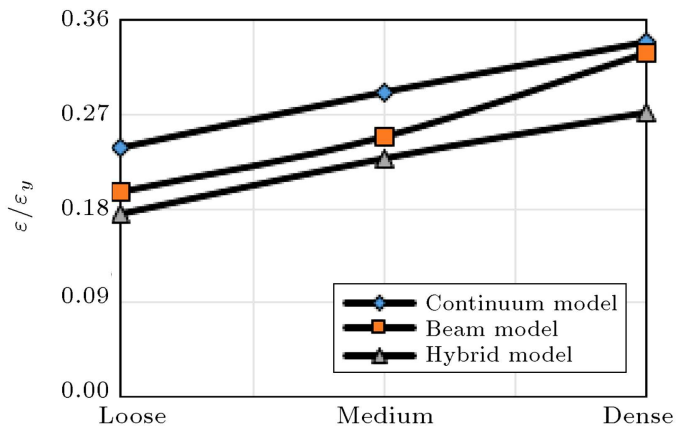

(a)

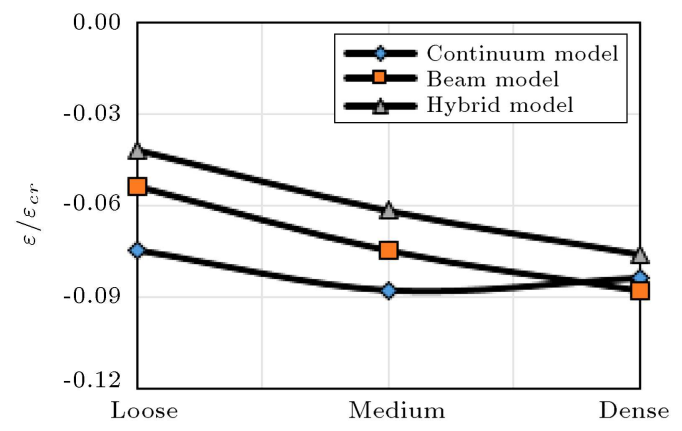

(b)

Figure 17. Comparison of maximum axial strain at a bend among continuum, beam, and hybrid models for the average of earthquakes ( $D=40 \mathrm{~cm}, t=9.5 \mathrm{~mm}, \theta=90^{\circ}$, horizontal propagation).

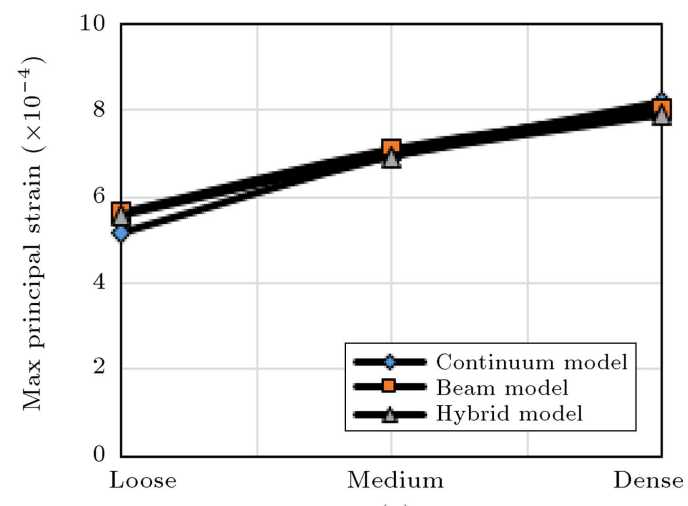

(a)

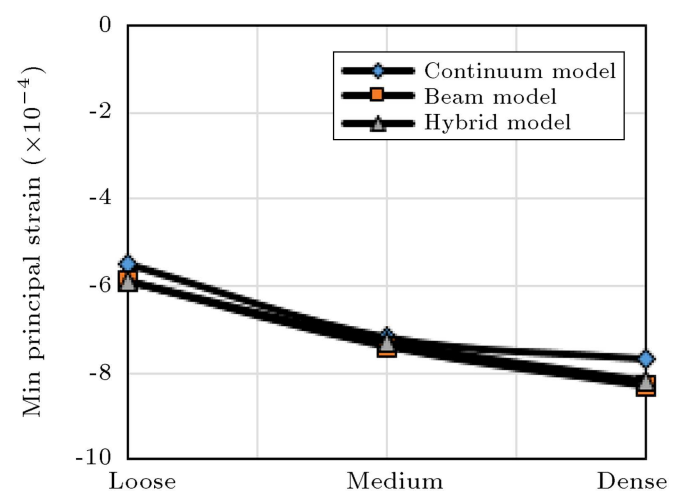

(b)

Figure 18. Comparison of principal strain at a bend between continuum, beam, and hybrid models for the average of earthquakes ( $D=40 \mathrm{~cm}, t=9.5 \mathrm{~mm}, \theta=90^{\circ}$, and horizontal propagation). 
strain at the bend for the average of the consistent earthquakes.

\section{A semi-analytical equation for the maximum axial strain}

The main objective of this study is to provide an equation for estimating the maximum axial strain of a buried pipe at its bend under propagating waves. According to Sections 4.5 and 4.6 , the maximum axial strain at a bend is a complex function of the elbow angle, soil stiffness (shear wave velocity), and $D / t$. Therefore, the following general equation is selected for calculating the maximum axial strain at bend (under horizontal propagation of waves) as an average of the earthquakes used in this study:

$$
\varepsilon=f(\theta) g\left(V_{s}, \theta\right) h\left(\frac{D}{t}, V_{s}\right),
$$

where $\varepsilon$ is the maximum axial strain, $f(\theta)$ function of the elbow angle $\theta, g\left(V_{s}, \theta\right)$ the bivariate function of the shear wave velocity of the surrounding soil $V_{s}$ and the elbow angle, and $h\left(\frac{D}{t}, V_{s}\right)$ is a bivariate function of $D / t$ and $V_{s}$. The reason why the functions $g$ and $h$ are of varying with two variables is that extent of the effect of $V_{s}$ and $\theta$ changes considerably and differently with the soil types investigated in this study based on the results of Sections 4.5 and 4.6. It should be noted that the above equation can only be used at a seismic level corresponding to earthquakes with a 475year return period in a highly seismic region associated with the design spectrum of Section 3.3. In addition, the burial depth of the pipe should be an ordinary value, i.e., about $1.5 \mathrm{~m}$ as assumed in this analysis. Because of the results presented in Sections 4.5 and 4.6, the above functions are taken to be different order polynomials of the mentioned variables. Constants of the polynomials are calculated by regression using the results of Sections 4.5 and 4.6 for the cases listed in Table 4 . There are 168 data points ( 24 cases multiplied by 7 earthquakes) for this regression analysis. The data points are shown in Figure 19.

Regression with the above data produces the following functions for the average of earthquakes:

$$
\begin{aligned}
f(\theta)= & .247\left(\frac{\theta}{\theta_{s}}\right)^{4}-3.791\left(\frac{\theta}{\theta_{s}}\right)^{3} \\
+ & 4.221\left(\frac{\theta}{\theta_{s}}\right)^{2}-2.045\left(\frac{\theta}{\theta_{s}}\right)+0.371 \\
g\left(V_{s}, \theta\right)= & \left(-0.214\left(\frac{\theta}{\theta_{s}}\right)+0.367\right)\left(\frac{V_{s}}{V_{s b}}\right)^{2} \\
& +\left(0.189\left(\frac{\theta}{\theta_{s}}\right)-0.138\right)\left(\frac{V_{s}}{V_{s b}}\right)
\end{aligned}
$$

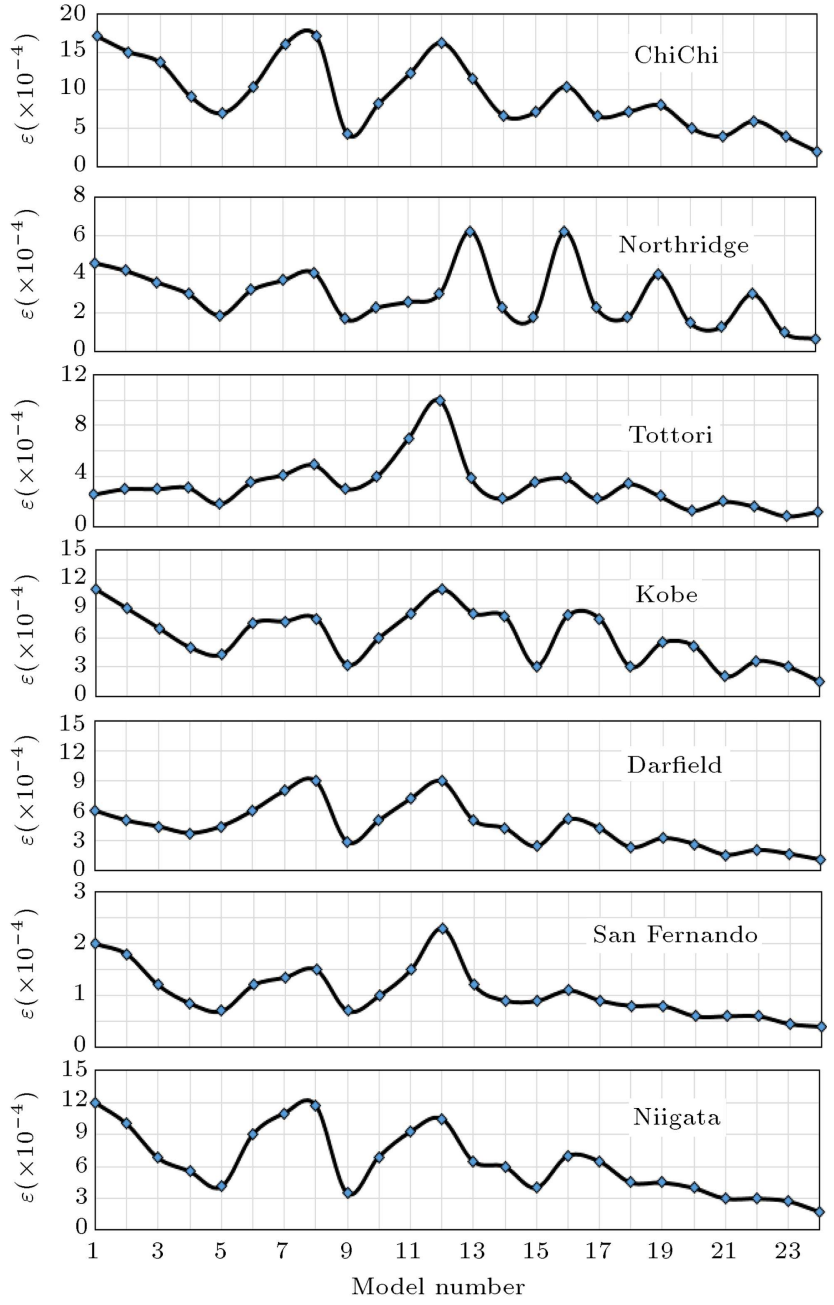

Figure 19. Collective results of maximum strain analysis.

$$
\begin{aligned}
+ & \left(-0.037\left(\frac{\theta}{\theta_{s}}\right)+0.053\right) \\
h\left(\frac{D}{t}, V_{s}\right)= & \left(-0.036\left(\frac{V_{s}}{V_{s b}}\right)^{2}-0.061\left(\frac{V_{s}}{V_{s b}}\right)\right. \\
& +0.042) \frac{D}{t}+\left(1.826\left(\frac{V_{s}}{V_{s b}}\right)+0.058\right)
\end{aligned}
$$

In Eq. (6), $\theta_{s}$ and $V_{s b}$ are a redundant angle equal to $180^{\circ}$ and a redundant shear wave velocity, respectively, in soil equal to $760 \mathrm{~m} / \mathrm{s}$. $\theta_{s}$ and $V_{s b}$ can be thought of as being the elbow angle for the straight pipe and the shear wave velocity in the bedrock, respectively. These parameters are only aimed at non-dimensionalizing the functions in Eq. (6) and making the numerical constants of this equation smoother.

To ensure a comparison, Eq. (5) is drawn in Figure 20 along with the maximum strains, given in Figure 19, averaged between seven earthquakes at each model number. Very good accuracy is observed.

For a straight pipe, ALA recommends calculating 


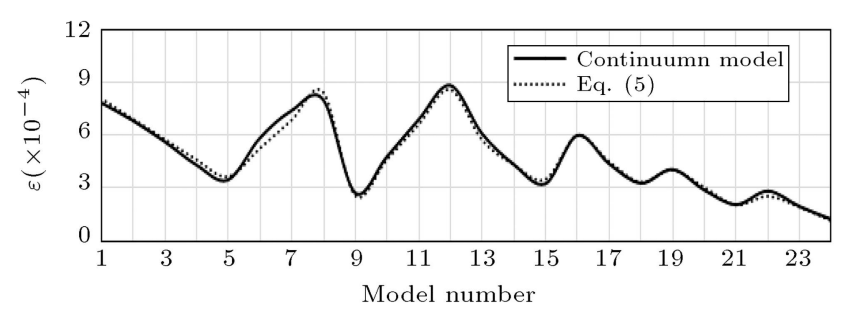

Figure 20. Comparison of maximum axial strain at a bend calculated using the continuum model and the proposed semi-analytical equation for the average of earthquakes.

the maximum normal strain under seismic activities using the following formula adopted from Newmark [1]:

$$
\varepsilon=\frac{V_{g}}{\alpha C_{s}}
$$

where $\varepsilon$ is the maximum axial strain, $V_{g}$ peak ground velocity generated by ground shaking, $C_{s}$ apparent propagation velocity for seismic waves (conservatively assumed to be 2 kilometers per second), and $\alpha$ equal to 2 .

Use of the above equation for Cases $22-24$ in Figure 20 generates strain values of $3.25 \times 10^{-4}, 1.97 \times$
$10^{-4}$, and $1.32 \times 10^{-4}$, respectively, that are consistent with the value predicted by Eq. (5) and Figure 20.

\section{The conversion factor}

For design applications, it is necessary that the maximum axial strain calculated by Eq. (5) be converted to the principal normal strain. The latter value can then be compared with the yield strain, perhaps with a safety factor, in a seismic pipe design. For this purpose, the ratios of the principal strain to the maximum axial strain (named conversion factor) are calculated. The elbow angle varies between $90^{\circ}-135^{\circ}$ and the average of maximum responses is calculated under horizontal propagation of waves for different soils and earthquakes, as shown in Figure 20. The average factor is the ratio of the maximum principal strain (averaged between earthquakes) to the maximum axial strain (again averaged between earthquakes). Also, for clarity purposes, the angle of orientation of the principal strain is also calculated. The results are shown in Tables 5 and 6 . It is interesting to note that how the variation of the results is small between different cases. This is such that the principal angle varies only between

Table 6. Ratio of the maximum principal strain to the maximum axial strain (the conversion factor) in the continuum model $(D=40 \mathrm{~cm}$ and $t=9.5 \mathrm{~mm})$.

\begin{tabular}{|c|c|c|c|c|}
\hline \multirow{2}{*}{$\begin{array}{c}\theta \\
(\mathrm{deg})\end{array}$} & \multirow[t]{2}{*}{ Earthquake } & \multicolumn{3}{|c|}{ Soil type } \\
\hline & & Loose sand & Medium sand & Dense sand \\
\hline \multirow{9}{*}{90} & San Fernando & 1.11 & 1.14 & 1.14 \\
\hline & Northridge & 1.13 & 1.16 & 1.14 \\
\hline & Kobe & 1.1 & 1.14 & 1.22 \\
\hline & ChiChi & 1.06 & 1.16 & 1.2 \\
\hline & Tottori & 1.05 & 1.17 & 1.3 \\
\hline & Darfield & 1.12 & 1.3 & 1.2 \\
\hline & Niigata & 1.08 & 1.19 & 1.15 \\
\hline & Average & 1.09 & 1.19 & 1.2 \\
\hline & Design factor & 1.1 & 1.2 & 1.2 \\
\hline \multirow{9}{*}{112.5} & San Fernando & 1.22 & 1.22 & 1.25 \\
\hline & Northridge & 1.16 & 1.17 & 1.05 \\
\hline & Kobe & 1.13 & 1.1 & 1.03 \\
\hline & ChiChi & 1.1 & 1.13 & 1.13 \\
\hline & Tottori & 1.14 & 1.13 & 1.04 \\
\hline & Darfield & 1.11 & 1.12 & 1.06 \\
\hline & Niigata & 1.13 & 1.14 & 1.14 \\
\hline & Average & 1.13 & 1.12 & 1.08 \\
\hline & Design factor & 1.1 & 1.1 & 1.1 \\
\hline \multirow{9}{*}{135} & San Fernando & 1.25 & 1.27 & 1.18 \\
\hline & Northridge & 1.22 & 1.13 & 1.04 \\
\hline & Kobe & 1.17 & 1.12 & 1.07 \\
\hline & ChiChi & 1.11 & 1.11 & 1.14 \\
\hline & Tottori & 1.2 & 1.23 & 1.05 \\
\hline & Darfield & 1.24 & 1.19 & 1.08 \\
\hline & Niigata & 1.17 & 1.18 & 1.09 \\
\hline & Average & 1.17 & 1.15 & 1.09 \\
\hline & Design factor & 1.2 & 1.2 & 1.1 \\
\hline
\end{tabular}


$37^{\circ}$ and $41^{\circ}$ and the average conversion factor changes between 1.1 and 1.2 for all soil types. Therefore, it can be concluded that the maximum axial strain possesses a prominent share of the principal strain. Furthermore, this factor is almost identical for different soils and different scaled earthquakes. As a result, to the safe side, the conversion factor can be considered to be 1.2 for the three sand types.

\section{Conclusions}

In this study, the maximum axial strain of a buried steel pipe was derived in the bend zone under seven different seismic excitations propagating in the vertical or horizontal directions. Then, a semi-analytical equation was determined to calculate the same quantity along with the principal strain at a bend with good accuracy in a much shorter time. The conclusions of this study are as follows:

1. The axial strains are much larger under horizontal propagation of seismic waves than the ones under vertical propagation;

2. While axial strains are larger in relatively more cases for the elbow angle of $90^{\circ}$, the maximum response does not differ much for the elbow angle range of 90-112.5 degrees under vertical propagation and for the range of 90-135 degrees under horizontal propagation of the seismic waves;

3. The diameter-to-thickness ratio is an impressive ratio for axial strain at a bend and its effect varies depending on the soil stiffness. In loose and medium sands, strain increases with increasing $D / t$; however, the course is reversed for the dense sand. For $D / t>50$ and $D / t<50$, the maximum axial strain is larger in stiffer and softer sands, respectively. At $D / t=50$, the maximum strain is almost the same in the case of different sands;

4. It was shown that use of the continuum model resulted in maximum axial strains at a bend which was to $30 \%$ larger than what was predicted by the beam or the hybrid models;

5. A semi-analytical formula was presented for calculating the maximum axial strain at a bend under an ensemble of earthquakes consistent with a design spectrum. It was shown that the equation estimated the response with very good accuracy;

6. A conversion factor was presented to convert the maximum axial strain to the principal strain at a bend. Value of the factor did not change much for different types of sand and varied between 1.1-1.2 for the average of earthquakes.

\section{Data availability statement}

Some or all data, models, or codes that support the findings of this study are available from the corresponding author upon reasonable request.

\section{References}

1. Newmark, N.M. "Problems in wave propagation in soil and rock", International Symposium on Wave Propagation and Dynamic Properties of Earth Materials, University of New Mexico, Albuquerque, pp. 7-26 (1968).

2. Sakurai, A. and Takahashi, T. "Dynamic stresses of underground pipelines during earthquake", Fourth World Conference on Earthquake Engineering, Santiago, Chile, pp. 81-95 (1969).

3. O'Rourke, M.J. and El Hmadi, K. "Analysis of continuous buried pipelines for seismic wave effects", Earthq. Eng. Struct. Dyn., 16, pp. 917-929 (1988).

4. Takada, S. and Tanabe K. "Three dimensional seismic response analysis of buried continuous or jointed pipelines", J. Press Vessel Technol., 109, pp. 80-87 (1987).

5. Takada, S. and Higashi, S. "Seismic response analysis for jointed buried pipeline by using shell FEM model", Tenth World Conference on Earthquake Engineering, pp. 5487-5492 (1992).

6. Stamos, A.A. and Beskos D.E. "Dynamic analysis of large 3-D underground structures by the BEM", Earthq. Eng. Struct. Dyn., 24, pp. 917-934 (1995).

7. Datta, T.K. "Seismic response of buried pipelines: a state-of-the-art review", Nucl. Eng. Des., 192, pp. 271-284 (1999).

8. Takada, S. and Katagiri, S. "Shell model response analysis of buried pipelines", Fourth U.S. Conference of Lifeline Earthquake Engineering, pp. 256-263 (1995).

9. Kouretzis, G.P., Bouckovalas, G.D., and Gantes C.J. "3-D shell analysis of cylindrical underground structures under seismic shear wave action", Soil Dyn. Earthq. Eng., 26, pp. 909-921 (2006).

10. Azizkandi, A.S., Baziar M.H., Modarresi, M., Salehzadeh. H., and Rasouli, H. "Centrifuge modeling of pile-soil-pile interaction considering relative density and toe condition", Scientia Iranica, A., 21(4), pp. 1330-1339 (2014).

11. Khaksar, R.Y., Moradi, M., and Ghalandarzadeh, A. "Response of buried oil and gas pipelines subjected to reverse faulting: A novel centrifuge-finite element approach", Scientia Iranica A., 25(5), pp. 2501-2516 (2018).

12. Zhou, X.P., Bi, J., and Qian, Q.H. "Numerical simulation of crack growth and coalescence in rock-like materials containing multiple pre-existing flaws", Rock Mechanics and Rock Engineering, 48(3), pp. 10971114 (2015). 
13. Bi, J., Zhou X.P., and Qian, Q.H. "The 3D numerical simulation for the propagation process of multiple pre-existing flaws in rock-like materials subjected to biaxial compressive loads", Rock Mechanics and Rock Engineering, 49(5), pp. 1611-1627 (2016).

14. Zhou, X.P. and Yang, H.Q. "Multiscale numerical modeling of propagation and coalescence of multiple cracks in rock masses", International Journal of Rock Mechanics and Mining Sciences, 55, pp. 15-27 (2012).

15. Zhou, X.P., Chen, J.W., and Berto, F. "XFEM based node scheme for the frictional contact crack problem", Computers and Structures, 231, 106221 (2020).

16. Chen, J.W. and Zhou, X.P. "The enhanced extended finite element method for the propagation of complex branched cracks", Engineering Analysis with Boundary Elements, 104, pp. 46-62 (2019).

17. Wang, Y., Zhou, X., and Xu, X. "Numerical simulation of propagation and coalescence of flaws in rock materials under compressive loads using the extended non-ordinary state-based peridynamics", Engineering Fracture Mechanics, 163, pp. 248-273 (2016).

18. Wang, Y., Zhou, X., Wang, Y., and Shou, Y. "A 3-D conjugated bond-pair-based peridynamic formulation for initiation and propagation of cracks in brittle solids", International Journal of Solids and Structures, 134, pp. 89-115 (2018).

19. Zhou, X., Wang, L., and Shou, Y. "Understanding the fracture mechanism of ring Brazilian disc specimens by the phase field method", International Journal of Fracture, 226(1), pp. 17-43 (2020).

20. Wang, L. and Zhou, X. "Phase field model for simulating the fracture behaviors of some disc-type specimens", Engineering Fracture Mechanics, 226, 106870 (2020).

21. Shah, H. and Chu, S. "Seismic analysis of underground structural element", J. Power Div., 100, pp. 53-62 (1974).

22. Ogawa, Y. and Koike, T. "Structural design of buried pipeline for sever earthquake", Soil Dyn. Earthq. Eng., 21, pp. 199-209 (2001).

23. Mclaughlin, P.M. and O'Rourke, M. "Strain in pipe elbows due to wave propagation hazard", Lifeline Earthquake Engineering in a Multihazard Environment, ASCE (2009).

24. Lee, D.H., Kim, B.H., Lee H., and Kong J.S. "Seismic behavior of a buried gas pipeline under earthquake excitations", Eng. Struct., 3, pp. 1011-1023 (2009).

25. Hatzigeorgiou, G.D. and Beskos, D.E. "Soil-structure interaction effects on seismic inelastic analysis of 3-D tunnels", Soil Dynamics and Earthquake Engineering, 30, pp. 851-861 (2010).

26. Saberi, M., Behnamfar, F., and Vafaeian, M. "A semianalytical model for estimating seismic behavior of buried steel pipes at bend point under propagating waves", Bull. Earthquake Eng., 11, pp. 1373-1402 (2013).
27. American Petroleum Institute "Specification for line pipe", API Specification 5L, Forty-Second Edition (2000).

28. Bowles, J.E., Foundation Analysis and Design, The McGraw-Hill Companies, N.Y. (1996).

29. Seed, H.B. and Idriss, I.M. "Soil moduli and damping factors for dynamic response analyses", Report No. EERC 70-10, Earthquake Engineering Research Center, University of California, Berkeley, C.A. (1970).

30. Jin, S., Li, Z., Dong, Z., Lan, T., and Gong, J. "A simplified fragility analysis methodology for containment structure subjected to overpressure condition", International Journal of Pressure Vessels and Piping, 184, 104104 (2020).

31. Wolf, J.P., Dynamic Soil-Structure Interaction, The Prentice-Hall Company (1985).

32. Liu, A.I., Hu, Y.X., Zhao, F.X., Li, X.J., Takada, S., and Zhao, L. "An equivalent-boundary method for the shell analysis of buried pipelines under fault movement", Acta Seismol. Sinica, 17, pp. 150-156 (2004).

33. PEER NGA, Pacific Earthquake Engineering Research Center, http://peer.berkeley.edu/, accessed July 2015.

34. SHAKE2000 "A Computer Program for the 1-D Analysis of Geotechnical Earthquake Engineering Problems", User's Manual, Geo Motions, LLC, Lacey, Wash., 2000, p. 264 (2015) http://www.geomotions.com/

35. ASCE7-10, Minimum Design Loads for Buildings and Other Structures, American Society of Civil Engineers (2010).

36. American lifelines alliance (ALA) Guidelines for the Design of Buried Steel Pipe, American Society of Civil Engineers (2001).

\section{Biographies}

Ehsan Mohammadi is a holder of MSc in Earthquake Engineering. He received his BS degree in Civil Engineering from the University of Shahrekord, Iran in 2013. He is a Professional Engineer and involves structural engineering profession. His specialty lies in supervising and designing civil engineering projects.

Farhad Behnamfar is an Associate Professor at the Department of Civil Engineering, Isfahan University of Technology, Isfahan, Iran. His expertise is Earthquake Engineering. He received his BSc in Civil Engineering and MSc in Structural Engineering both from Tehran University in 1988 and 1990, respectively, and $\mathrm{PhD}$ in Earthquake Engineering from Tohoku University, Japan, 1997. His PhD thesis was on structure-soilstructure interaction under spatially variable earthquake ground motion. 